\title{
Directed Ligand Exchange on the Surface of PbS Nanocrystals: Implications for Incoherent Photon Conversion
}

\author{
Philippe B. Green, Francisco Yarur Villanueva, Christian J. Imperiale, Minhal Hasham, \\ Karl Z. Demmans, Darcy C. Burns and Mark W.B. Wilson* \\ Department of Chemistry, University of Toronto \\ Toronto, Ontario M5S3H6, Canada
}

\section{Contents}

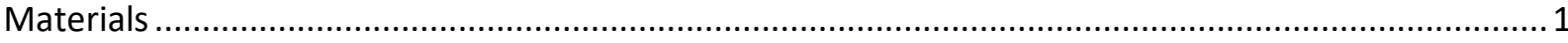

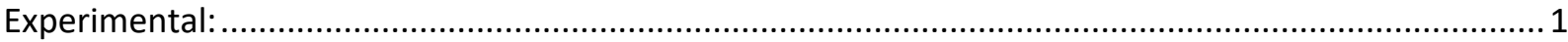

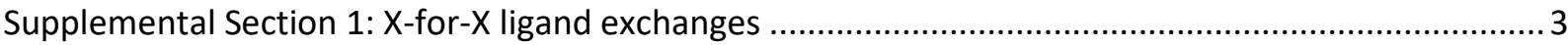

Supplemental Section 2: Full data sets for L-type promoted Z-type ligand displacement....................10

Comment on the vinylic proton linewidths during L-promoted Z-type ligand displacement ............16

Supplemental Section 3: Estimation of the concentration of bound 9-ACA .....................................17

Supplemental Section 4: Data fitting and extra characterization of the exchanged NCs.....................21

Supplemental Section 5 : UDA exchanged Z-type ligand displacement ...........................................2 27

Supplemental Section 6: Variable temperature ${ }^{1} \mathrm{H}$ NMR measurements: .........................................28

Supplemental Section 7: Homogeneous linewidth and T2 measurements:.....................................30

Supplemental Section 8: Transmission Electron Microscopy characterization .....................................38

Supplemental Section 9: X-for-X exchanges with Entropic Ligands................................................... 40

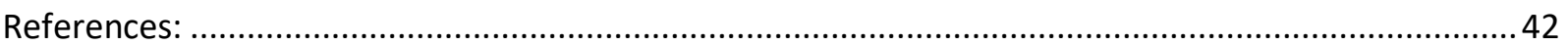

\section{Materials}

Lead (II) Oxide (Sigma Aldrich 99.999\%), Hexamethyldisilathiane (TMS-S, Sigma Aldrich), Oleic acid (OA, Sigma Aldrich 90\%), 1-Octadecene (ODE, Sigma Aldrich 90\%), undec-10-enoic acid (UDA, Sigma Aldrich 98\%), 9-anthracene carboxylic acid (9-ACA, Sigma Aldrich 99\%), 2-hexyldecanoic acid (2HDA, Sigma Aldrich, 96\%), dicyclohexylacetic acid (DCHAA, Sigma Aldrich, 99\%) benzene-d6 (Sigma Aldrich 99.6\%), acetone (Sigma Aldrich 99.6\%), tetrachloroethylene (TCE, Sigma Aldrich 90\%), and hexanes (Sigma Aldrich 99\%) were used without further purification.

\section{Experimental:}

\section{Nanocrystal synthesis:}

The reaction was performed as we have previously presented. ${ }^{1-3}$ The lead solution was prepared by adding $0.446 \mathrm{~g}$ ( $2 \mathrm{mmol}$ ) of lead oxide, $3.8 \mathrm{~mL} \mathrm{OA}(3.4 \mathrm{~g}, 12 \mathrm{mmol}$ ) and $25 \mathrm{~mL}$ ODE in a three-neck roundbottom flask. The solution was placed under vacuum, heated to $120^{\circ} \mathrm{C}$, and vigorously stirred for at least 
30 minutes, until all the lead oxide dissolved. Solutions took on a pale-yellow colour. The solution was then set to $90^{\circ} \mathrm{C}$ and left to allow the temperature to stabilize. The TMS-S solution was prepared by adding $0.06 \mathrm{~mL}(0.28 \mathrm{mmol})$ of TMS-S to $1.5 \mathrm{~mL}$ of ODE in a nitrogen glovebox and then loading the mixture into a syringe. Once the desired temperature of the lead solution was reached, the TMS-S solution was rapidly injected. The reaction was allowed to proceed for 7 minutes before quenching with an ice bath.

Purification was performed by four successive cycles of antisolvent precipitation and redispersion. Acetone was used as the antisolvent for all purification steps and hexanes was used to redisperse the nanocrystals, with the exception of the last redispersion which was in either in TCE or benzene-d6 for optical spectroscopy or ${ }^{1} \mathrm{H} N \mathrm{NR}$, respectively. The precipitation was performed by centrifugation for $80 \mathrm{~s}$ at $6000 \mathrm{rpm}$ (4430 rcf).

\section{Ligand exchange:}

Stock solutions of $10 \mathrm{mg} / \mathrm{ml}$ of UDA and 9-ACA were prepared in benzene-d6, and varying quantities (See e.g. Fig S3 for amounts for a typical X-for-X exchange) added to NC solutions (50-80uM, calculated from optical absorption ${ }^{4}$ ). The solution was then stirred for approximately 5 minutes. For purification, twice the volume of acetone was added, and the nanocrystals were precipitated by centrifugation for $80 \mathrm{~s}$ at 6000 rpm (4430 rcf). The supernatant was discarded, and the nanocrystals were dispersed in hexanes. This purification step was then performed again, with the second redispersion in benzene-d6. All ligand exchanges were performed in a nitrogen-filled glovebox.

\section{Characterization:}

Optical absorption spectra were taken on a Perkin Elmer Lambda 1050 UV/Vis spectrophotometer. Transmission electron microscopy was performed using a Hitachi HT7700 microscope. Samples for TEM were prepared by drop-casting nanoparticles in hexanes onto a carbon grid. Measurements to allow the estimation of the size distribution were all performed using 50,000x magnification. The TEM image was first binarized. If contrast was weak, NCs were manually picked out from background noise, and NCs with an especially unusual appearance (i.e. aggregates, or the result of local layering) were excluded. Otherwise, the binarized images were used directly. This generated image files with at least 680 distinct particles from which size distributions were extracted using ImageJ, converting the measured area into an effective diameter assuming a spherical nanocrystal.

Nuclear magnetic resonance was performed on either a $400 \mathrm{MHz}$ Varian MercuryPlus NMR spectrometer or a $500 \mathrm{MHz}$ Agilent DD2 NMR spectrometer. Titrations with either X-type ligands or TMEDA and variabletemperature measurements were performed on the $400 \mathrm{MHz}$ instrument, while CPMG and DOSY measurements were performed on the $500 \mathrm{MHz}$ machine. For $\mathrm{X}$-for-X exchanges, we waited ten minutes after ligand addition before taking the first measurement. For L-promoted Z-type ligand removal, we waited 30 minutes after TMEDA addition before taking the first spectrum. Every spectrum was taken with $25 \mathrm{~s}$ delay times and either 8 or 16 scans. 


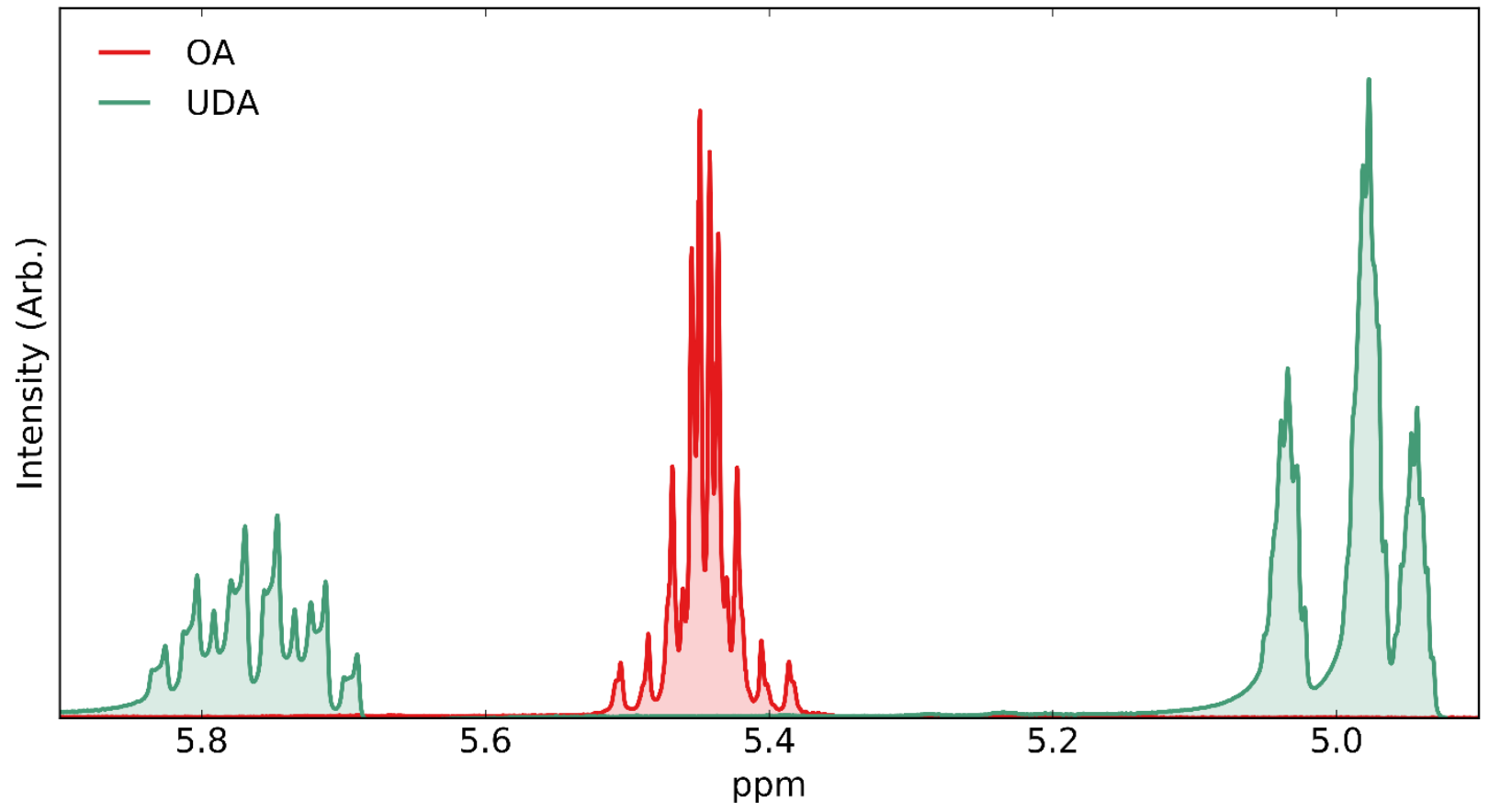

Figure S1: $\mathrm{H}^{1}$ NMR spectra of the vinylic region of our stock solutions of free OA (red) and UDA (green) in benzene-d6. The UDA resonance around $5.0 \mathrm{ppm}$ corresponds to an integration of two, while the peak around $5.8 \mathrm{ppm}$ has an integration of one. The OA peak has an integration of two. The spectral difference enables simultaneous quantification and identification of OA and UDA. ${ }^{5}$ 


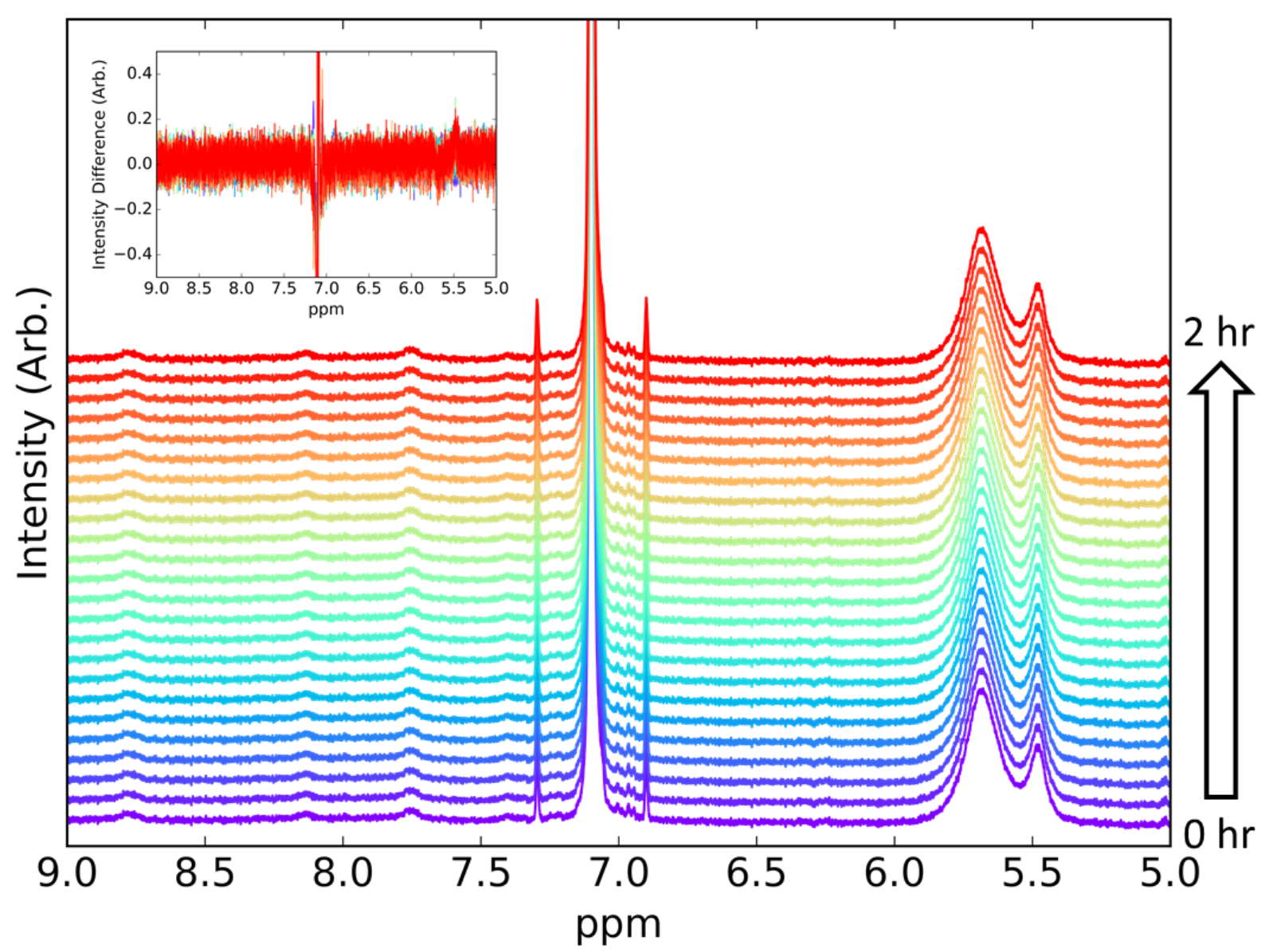

Figure S2: Two-hour scan with five-minute intervals of $X$-for-X exchange with 9-ACA. Free 9-ACA resonances can be observed around 7.76, 8.13 and $8.77 \mathrm{ppm}$. Resonances from bound 9-ACA are not directly observable in these spectrum, but its presence can be inferred by the displaced OA-seen as the sharp resonance at $5.47 \mathrm{ppm}$ (as opposed to bound OA at $5.67 \mathrm{ppm}$.) Importantly, the spectra do not evolve over time, indicating that equilibrium following the addition of 9-ACA addition is reached rapidly ( $<5$ minutes). This is our general observation for $X$ for $X$ exchanges in good solvents. The inset overlays the difference between all spectra and the first spectrum to emphasize that no evolution in features is observed. 


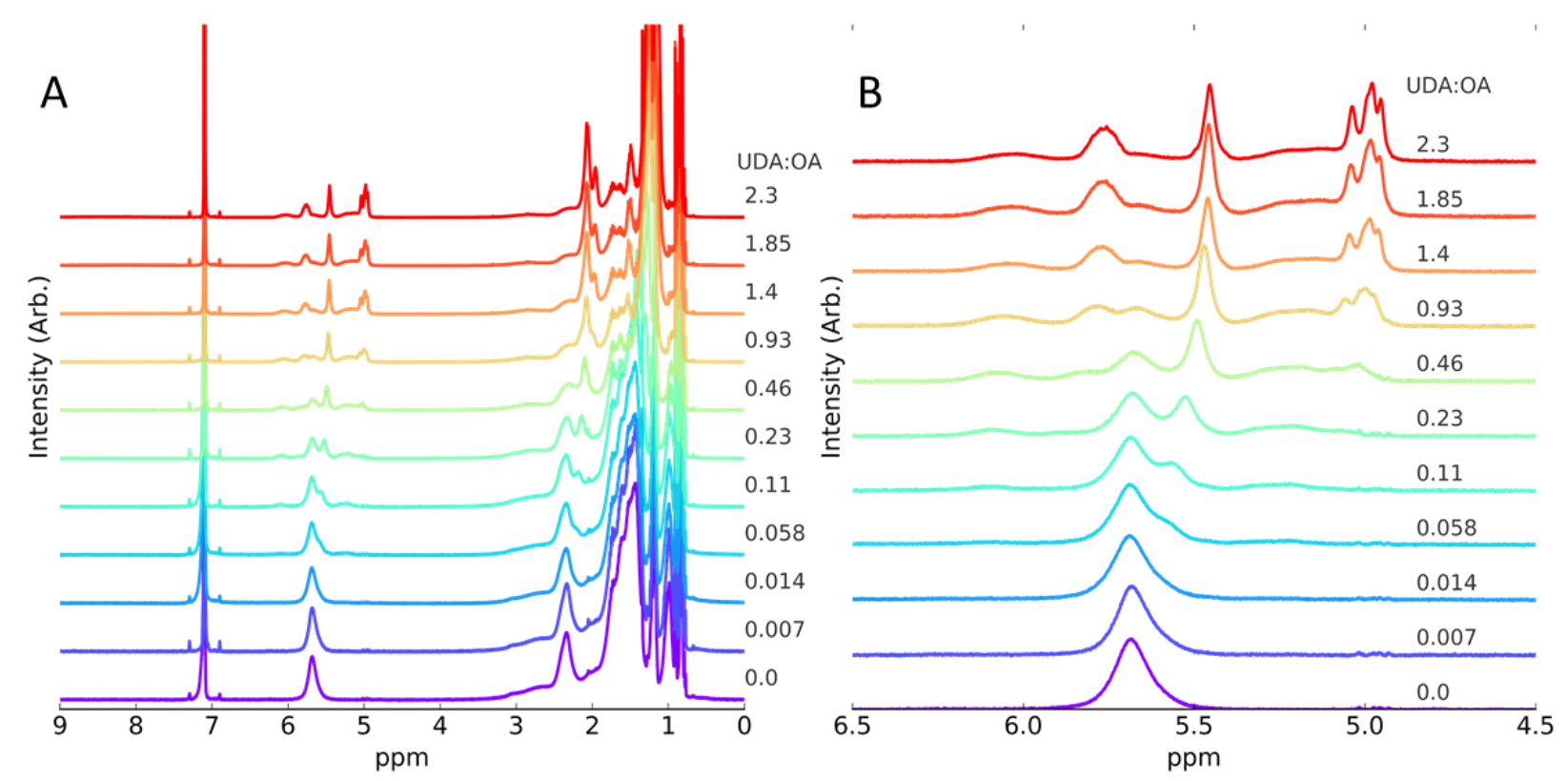

Figure S3: X-for-X exchange with UDA. Starting from a $550 \mu \mathrm{L}$ solution of nanocrystals ( $80 \mu \mathrm{M}$ in benzened6), we progressively increase the concentration of UDA by titrating from a stock UDA solution $(10 \mathrm{mg} / \mathrm{mL})$. A) Full spectrum throughout the exchange. B) Zooming in on the vinylic peaks of $O A$, the initial peak near $5.7 \mathrm{ppm}$ is due to the bound vinylic protons of OA. As UDA is titrated, several new peaks appear: Free $O A$ around $5.5 \mathrm{ppm}$, free UDA at 5 at $5.8 \mathrm{ppm}$ and bound UDA at 5.3 and $6.1 \mathrm{ppm}$. We observed significant displacement of OA by UDA (>90\%). However, little broadening of bound OA features are observed at any point during this exchange. (c.f. Figure $\mathrm{S} 4$ ) 


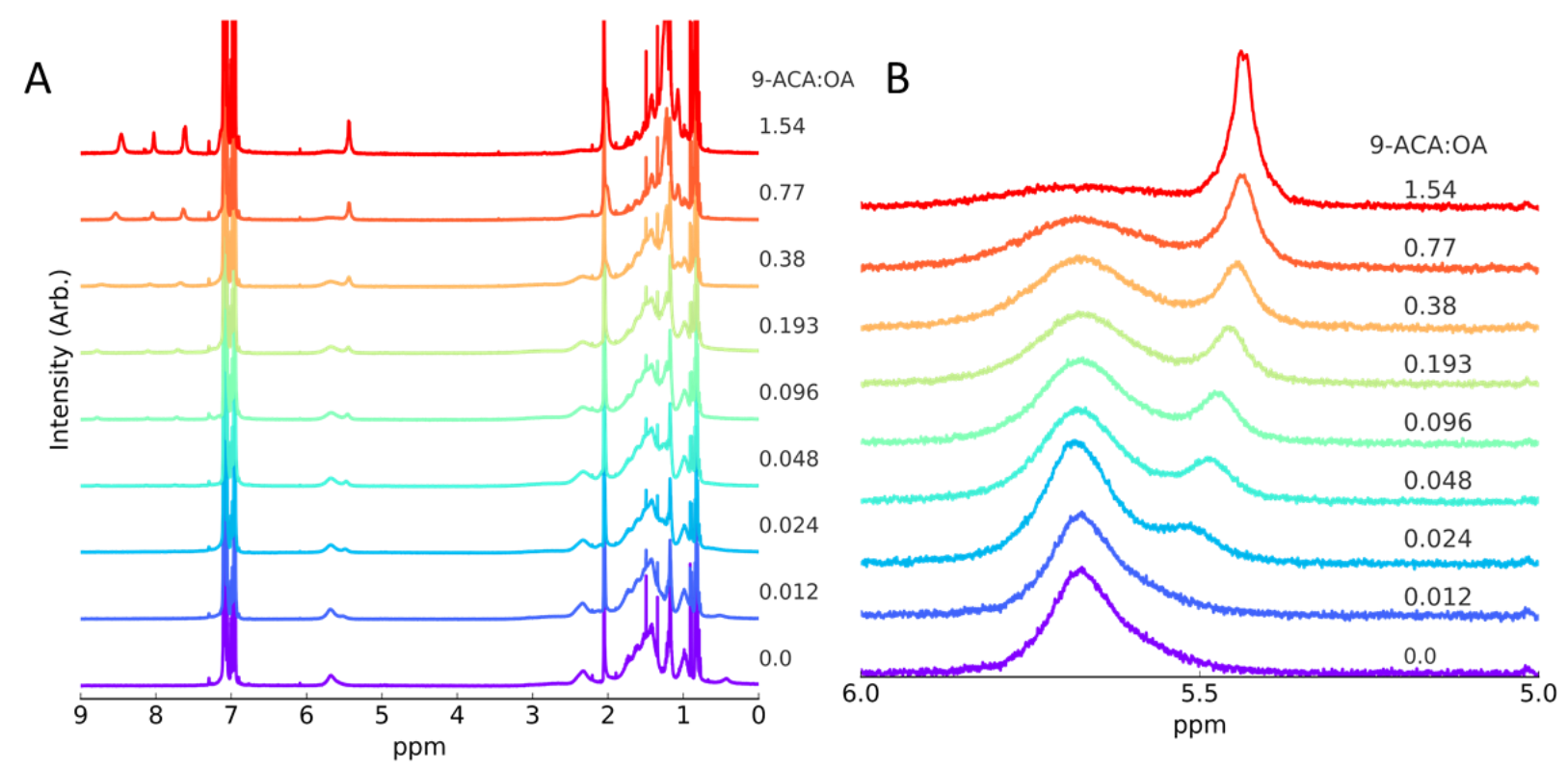

Figure S4: X-for-X exchange with 9-ACA. Starting from $550 \mu \mathrm{L}$ solution of nanocrystals ( $80 \mu \mathrm{M}$ in benzened6), we progressively increase the concentration of 9-ACA by titrating from a stock 9-ACA solution $(10 \mathrm{mg} / \mathrm{ml})$. A) Full spectrum throughout the exchange. B) Zooming in on the vinylic peaks of $O A$, the initial peak is due to the bound vinylic protons of OA. (N.B. the differing horizontal scale from Figure S3-the widths of these features are equivalent prior to ligand exchange.) As 9-ACA is progressively added, several new peaks appear: Free OA around $5.5 \mathrm{ppm}$ and free $9-\mathrm{ACA}$ at $7.76,8.13$ and $8.77 \mathrm{ppm}$. Importantly we cannot discern features corresponding to bound 9-ACA in this experiment. (c.f. Figure S5) In addition, the bound $\mathrm{OA}$ feature around $5.7 \mathrm{ppm}$ undergoes a significant broadening through the titration. (c.f. Figure S3) 


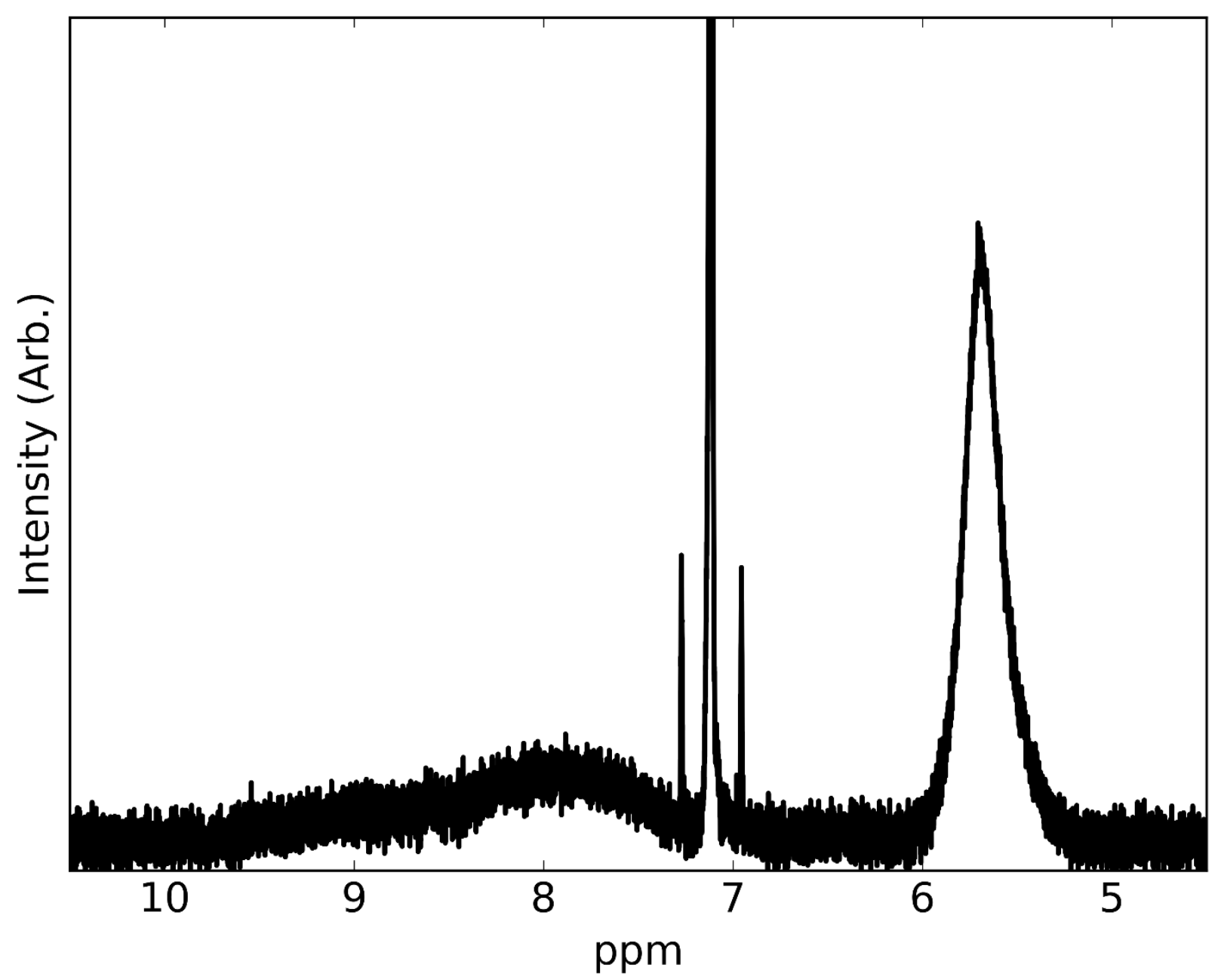

Figure S5: ${ }^{1} \mathrm{H}$ spectra of mixed 9-ACA/OA nanocrystals taken using a $500 \mathrm{MHz}$ instrument. The peak around $5.70 \mathrm{ppm}$ corresponds to the vinylic protons of the remaining OA. The peak at $7.11 \mathrm{ppm}$ comes from solvent impurities. The broad feature from 7-10 ppm is associated with bound 9-ACA. This peak is a convolution of the 5 distinct resonances expected in free 9-ACA. (Including the three features clearly discernable in free 9-ACA (Figure S2), as well as two additional resonances near the solvent peaks) The extreme broadening of the ${ }^{1} \mathrm{H}$ resonances of bound 9-ACA due to its close proximity to the large nanocrystal significantly impedes the direct quantitative determination of it concentration. 

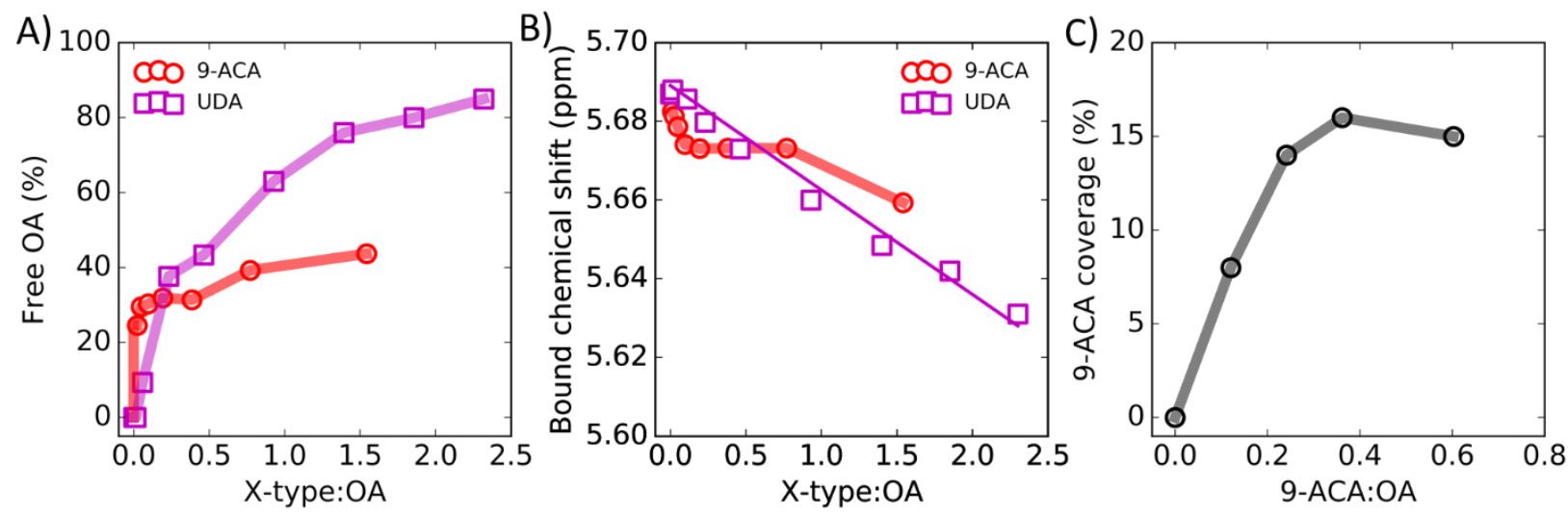

Figure S6:

A) Tracking the percentage of displaced $\mathrm{OA}$ by ${ }^{1} \mathrm{H}$ NMR with added equivalents of either UDA or 9-ACA. Importantly, comparing the $X$-for-X exchanges between UDA and 9-ACA reveals distinct exchange efficiencies: the addition of 2.4 equivalents of UDA (UDA:OA) efficiently replaces the majority of native OA, consistent with previous reports. ${ }^{5}$ In contrast, the 9-ACA exchange appears limited and plateaus at about $40 \%$ OA displacement.

B) Peak position of the bound OA vinylic protons throughout the X-for-X exchanges with both UDA and 9ACA. The peak position is a function of the surrounding chemical environment. For UDA exchanges, we observe a linear chemical shift with concentration, suggesting a continuous change in chemical environment throughout the titration. In contrast, 9-ACA exchanges result in an initial decrease followed by a plateau, suggesting a halting of the ligand exchange. These trends are qualitatively similar to the parallel displacement of OA (panel A), and are consistent with previous observations that exchanges with bulky ligands are hindered at some fraction of the available binding sites. (e.g. Ref. ${ }^{6}$ ) We also observe a concomitant, massive broadening of the bound OA peak as more 9-ACA is introduced (Figure S4B).

C) Progress of the 9-ACA coverage achieved by adding our stock 9-ACA solution $(10 \mathrm{mg} / \mathrm{mL}$ in benzened6) to $550 \mu \mathrm{L}$ of $80 \mu \mathrm{M}$ PbS NCs in the same solvent. Due to the poor visibility of bound 9-ACA in NMR (Figure S5), the coverage was determined by subsequent L-promoted removal. (See supplementary section 3 for calculations) Consistently, throughout the experiment, we could not reach 9-ACA coverages higher then $16 \%$ following purification, even though the OA displacement in A) suggests that a $40 \%$ 9-ACA coverage is possible. This apparent effect could be an artifact of the reduction of colloidal stability of NCs with high 9-ACA coverage due to the poor solubility of these ligands in deuterated benzene, because aggregation or precipitation could cause the ${ }^{1} \mathrm{H}$ resonances of bound $\mathrm{OA}$ ligands to become undetectably broad, skewing the calculation of fractional coverage. This behaviour is in contrast to UDA exchanges, which maintain high coverages ( $>90 \%)$, even after purification.

Lastly, we note that the relatively low X:OA equivalents required to drive either the UDA and 9-ACA exchanges towards their endpoint suggest that both ligands experience some kind of favourable intermolecular interaction at the surface-especially given the strong chemical similarities of UDA and OA. Similarly effective exchanges are usually observed with ligands with elevated binding strengths such as phosphonates ${ }^{7,8}$, but have also been observed if intermolecular interactions are present. ${ }^{9,10}$ 


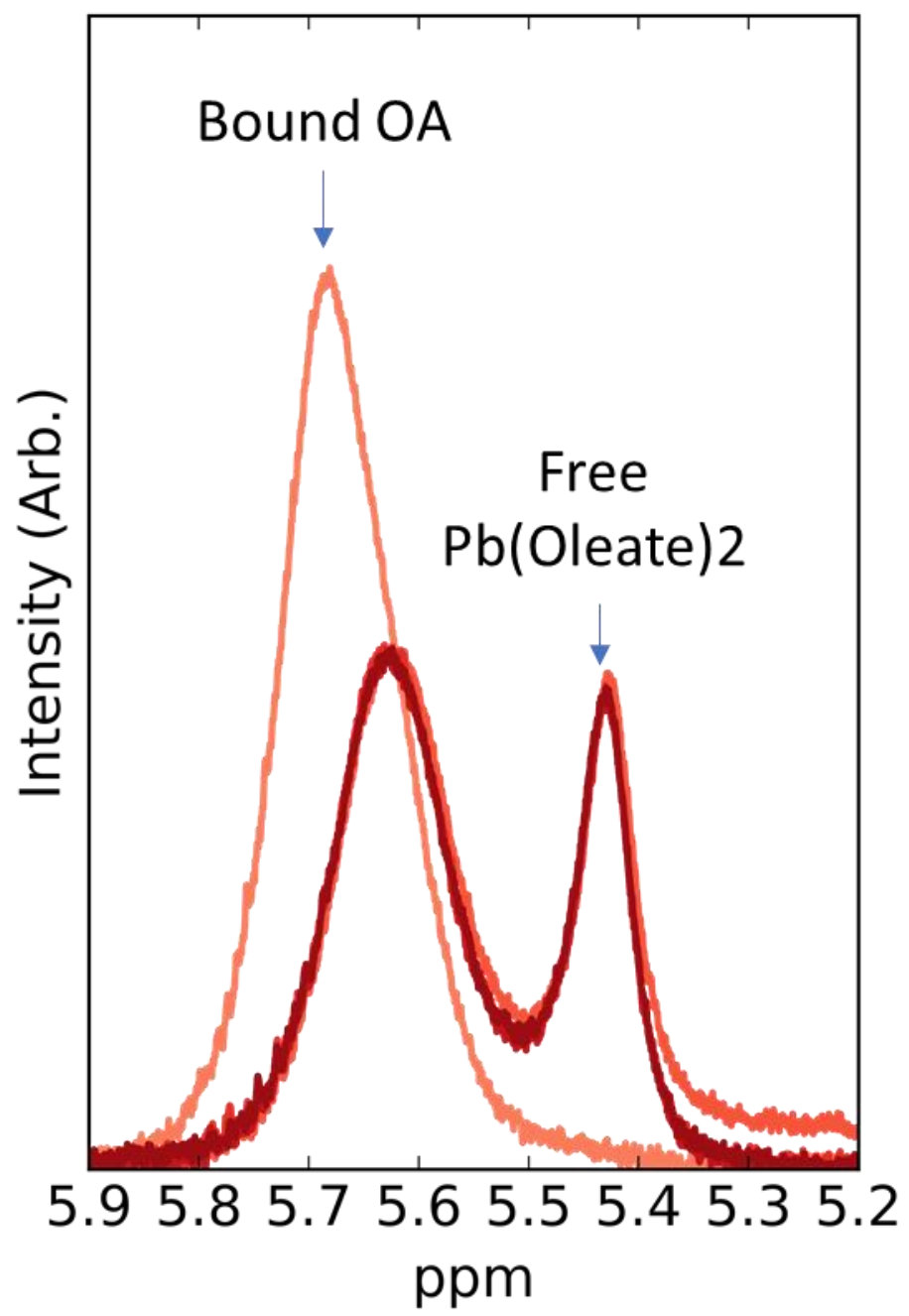

Figure S7: L-promoted Z-type ligand displacement with TMEDA reaches equilibrium by 30 minutes after addition. Initial NMR spectra of bound OA shows only a broad peak around 5.7 ppm (light red). As TMEDA is introduced, free $O A$ is observed to appear around $5.4 \mathrm{ppm}$. The three increasingly dark red spectra were taken after sequential 30-minute intervals. No significant difference is observed after the first 30 minutes suggesting that equilibrium is reached within this time. 

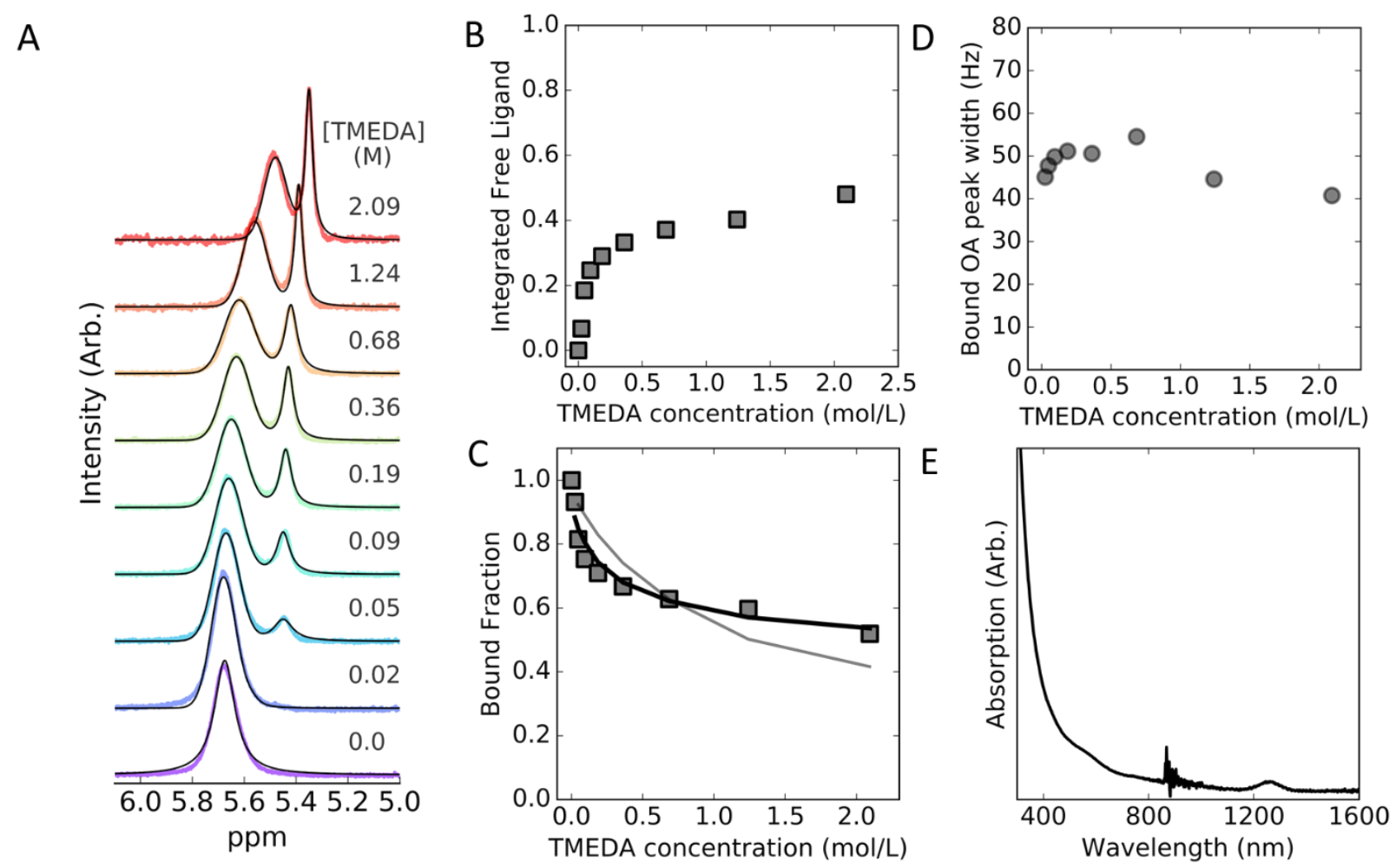

Figure S8: Full data set for L-type promoted Z-type ligand displacement for unexchanged NCs (OA only). A) ${ }^{1} \mathrm{H}$ NMR spectra of the resonances for free and bound OA from solutions of OA-only NCs to which increasing amounts of TMEDA are added.

B) Integrated area of the free OA ligands normalized to the total OA signal (free+bound).

C) Displacement isotherms for OA. The darker solid line corresponds to a two-equilibrium-constant fit, while the lighter line is a model for a single-equilibrium process.

D) The width of the bound OA peak is observed to slightly increase initially, and then to slowly decrease.

E) Optical absorption spectra of the unexchanged NC. The noise near $900 \mathrm{~nm}$ is an artifact resulting from insufficient detector sensitivity. 

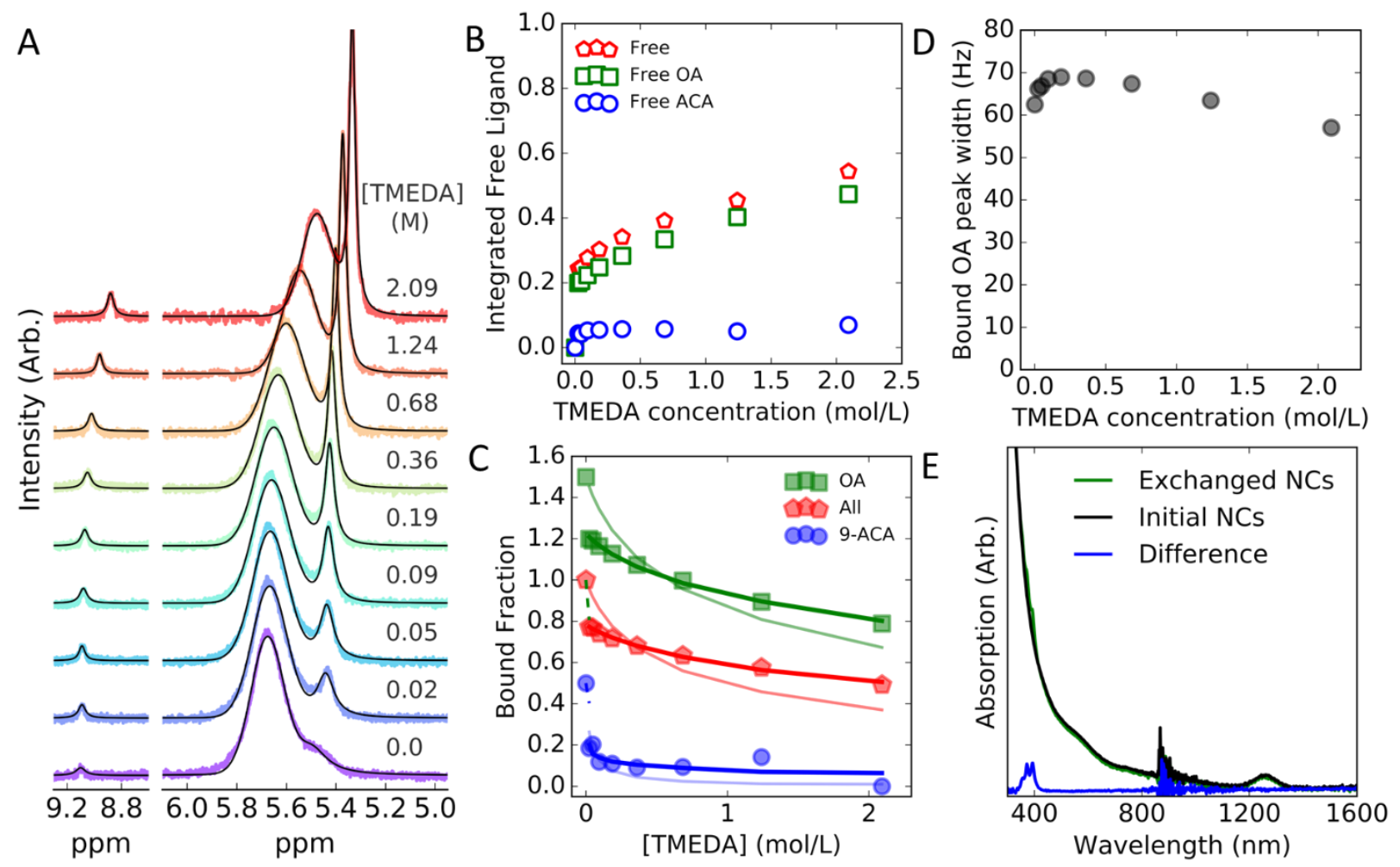

Figure S9: Full data set for L-type promoted Z-type ligand displacement for 9-ACA exchanged NCs, with an 8\% 9-ACA coverage as determined by NMR. (See supplementary section 3 )

A) ${ }^{1} \mathrm{H}$ NMR spectra of the resonances for free 9-ACA (left), and free and bound OA (right) from solutions of mixed 9-ACA/OA NCs to which increasing amounts of TMEDA are added.

B) Integrated area of the free OA (green squares), free 9-ACA (blue circles) and their sum (red pentagons). C) Displacement isotherms for OA (green square), free 9-ACA (blue circles) and their sum (red pentagons). The darker solid line corresponds to a two-equilibrium-constant fit, while the lighter line is a model for a single-equilibrium process. The respective isotherms are offset for clarity.

D) The width of the bound OA peak is observed to slightly increase initially, and then to slowly decrease.

E) Absorption spectra of the exchanged NC. We extract the isolated absorption of 9-ACA by taking the difference (blue line) between the absorption of the exchanged (green line) and unexchanged (black line) solutions. Using an absorption coefficient of $9700 \mathrm{~cm}^{-1} \mathrm{M}^{-1}$ at the peak $(\lambda: 380 \mathrm{~nm})$, and the known relations between optical density, size, and concentration for $\mathrm{PbS} \mathrm{NCs},{ }^{4}$ we estimate that there are 35 9-ACA per NC in this solution. 

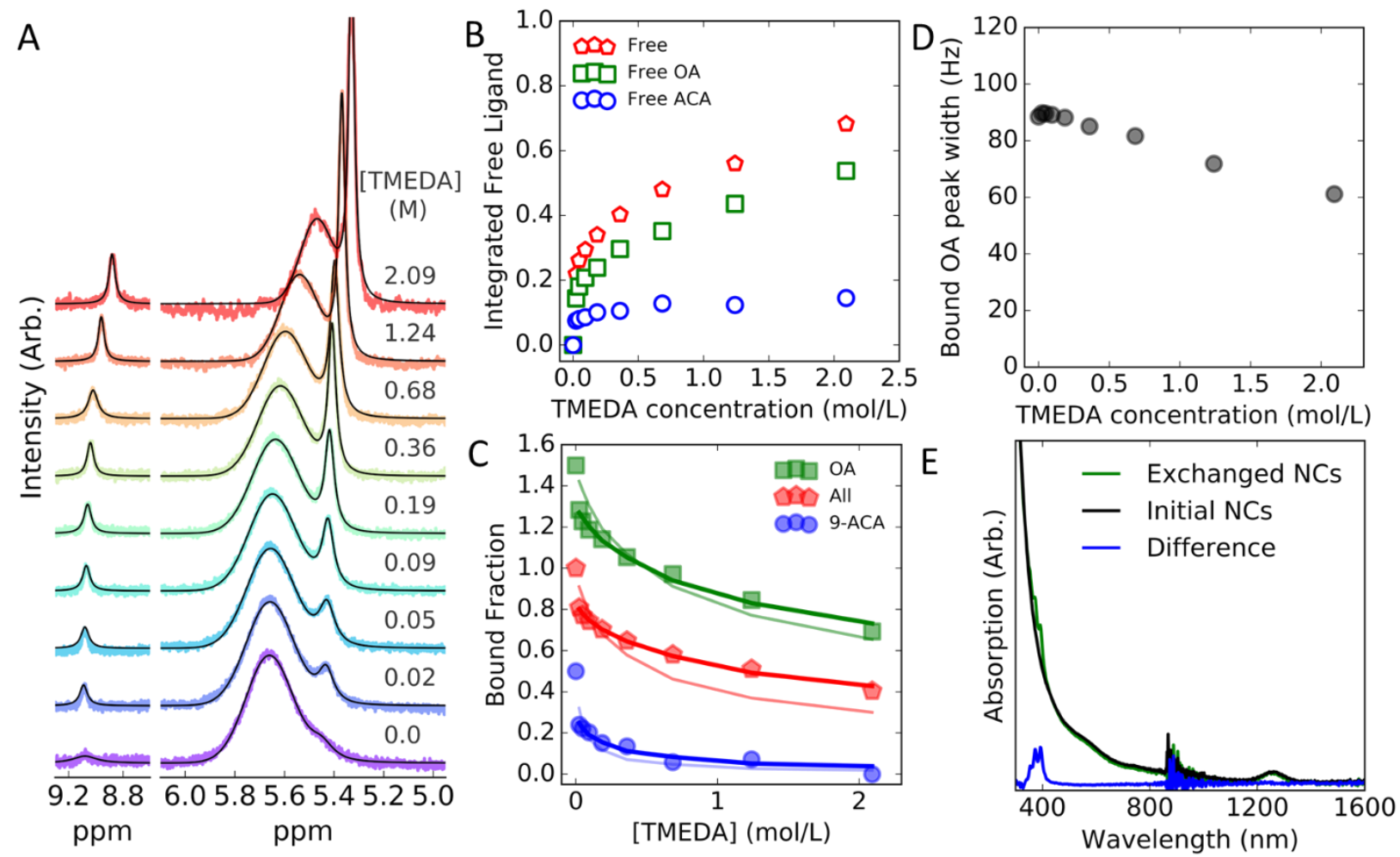

Figure S10: Full data set for L-type promoted Z-type ligand displacement for 9-ACA exchanged NCs, with an 14\% 9-ACA coverage as determined by NMR. (See supplementary section 3)

A) ${ }^{1} \mathrm{H}$ NMR spectra of the resonances for free 9-ACA (left), and free and bound OA (right) from solutions of mixed 9-ACA/OA NCs to which increasing amounts of TMEDA are added.

B) Integrated area of the free OA (green squares), free 9-ACA (blue circles) and their sum (red pentagons). C) Displacement isotherms for OA (green square), free 9-ACA (blue circles) and their sum (red pentagons). The darker solid line corresponds to a two-equilibrium-constant fit, while the lighter line is a model for a single-equilibrium process. The respective isotherms are offset for clarity.

D) The width of the bound OA peak is observed to slightly increase initially, and then to slowly decrease.

E) Absorption spectra of the exchanged NC. We extract the isolated absorption of 9-ACA by taking the difference (blue line) between the absorption of the exchanged (green line) and unexchanged (black line) solutions. Using an absorption coefficient of $9700 \mathrm{~cm}^{-1} \mathrm{M}^{-1}$ at the peak $(\lambda: 380 \mathrm{~nm})$ and the known relations between optical density, size, and concentration for $\mathrm{PbS} \mathrm{NCs},{ }^{4}$ we estimate that there are 63 9-ACA per $\mathrm{NC}$ in this solution. 

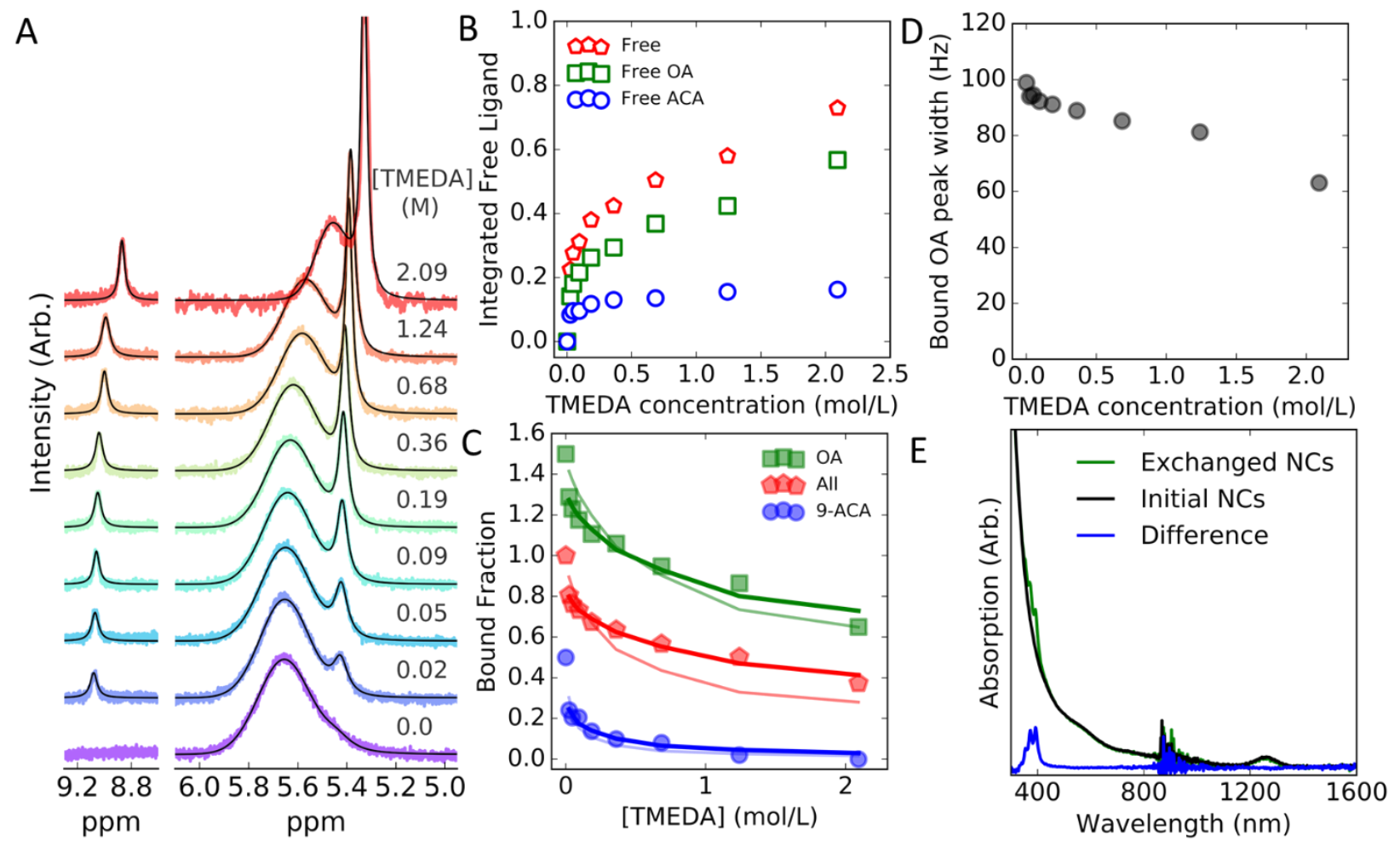

Figure S11: Full data set for L-type promoted Z-type ligand displacement for 9-ACA exchanged NCs, with an 16\% 9-ACA coverage as determined by NMR. (See supplementary section 3 )

A) ${ }^{1} \mathrm{H}$ NMR spectra of the resonances for free 9-ACA (left), and free and bound OA (right) from solutions of mixed 9-ACA/OA NCs to which increasing amounts of TMEDA are added.

B) Integrated area of the free OA (green squares), free 9-ACA (blue circles) and their sum (red pentagons). C) Displacement isotherms for OA (green square), free 9-ACA (blue circles) and their sum (red pentagons). The darker solid line corresponds to a two-equilibrium-constant fit, while the lighter line is a model for a single-equilibrium process. The respective isotherms are offset for clarity.

D) The width of the bound OA peak is observed to strictly decrease.

E) Absorption spectra of the exchanged NC. We extract the isolated absorption of 9-ACA by taking the difference (blue line) between the absorption of the exchanged (green line) and unexchanged (black line) solutions. Using an absorption coefficient of $9700 \mathrm{~cm}^{-1} \mathrm{M}^{-1}$ at the peak $(\lambda: 380 \mathrm{~nm})$ and the known relations between optical density, size, and concentration for $\mathrm{PbS} \mathrm{NCs},{ }^{4}$ we estimate that there are 74 9-ACA per NC in this solution. 

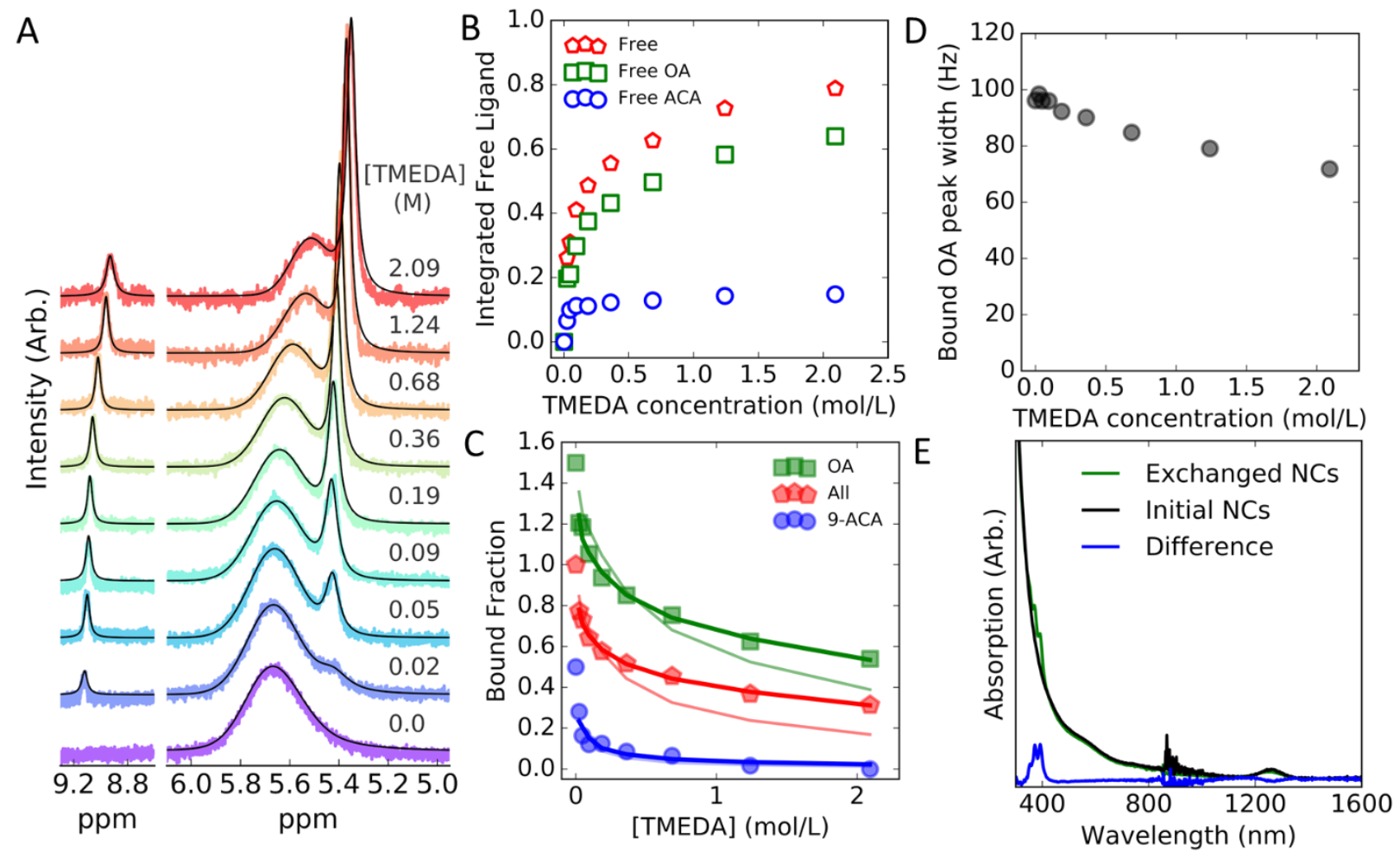

Figure S12: Full data set for L-type promoted Z-type ligand displacement for 9-ACA exchanged NCs, with an 15\% 9-ACA coverage as determined by NMR. (See supplementary section 3 )

A) ${ }^{1} \mathrm{H}$ NMR spectra of the resonances for free 9-ACA (left), and free and bound OA (right) from solutions of mixed 9-ACA/OA NCs to which increasing amounts of TMEDA are added.

B) Integrated area of the free OA (green squares), free 9-ACA (blue circles) and their sum (red pentagons). C) Displacement isotherms for OA (green square), free 9-ACA (blue circles) and their sum (red pentagons). The darker solid line corresponds to a two-equilibrium-constant fit, while the lighter line is a model for a single-equilibrium process. The respective isotherms are offset for clarity.

D) The width of the bound OA peak is observed to strictly decrease.

E) Absorption spectra of the exchanged NC. We extract the isolated absorption of 9-ACA by taking the difference (blue line) between the absorption of the exchanged (green line) and unexchanged (black line) solutions. Using an absorption coefficient of $9700 \mathrm{~cm}^{-1} \mathrm{M}^{-1}$ at the peak $(\lambda: 380 \mathrm{~nm})$ and the known relations between optical density, size, and concentration for $\mathrm{PbS} \mathrm{NCs},{ }^{4}$ we estimate that there are 68 9-ACA per NC in this solution. 


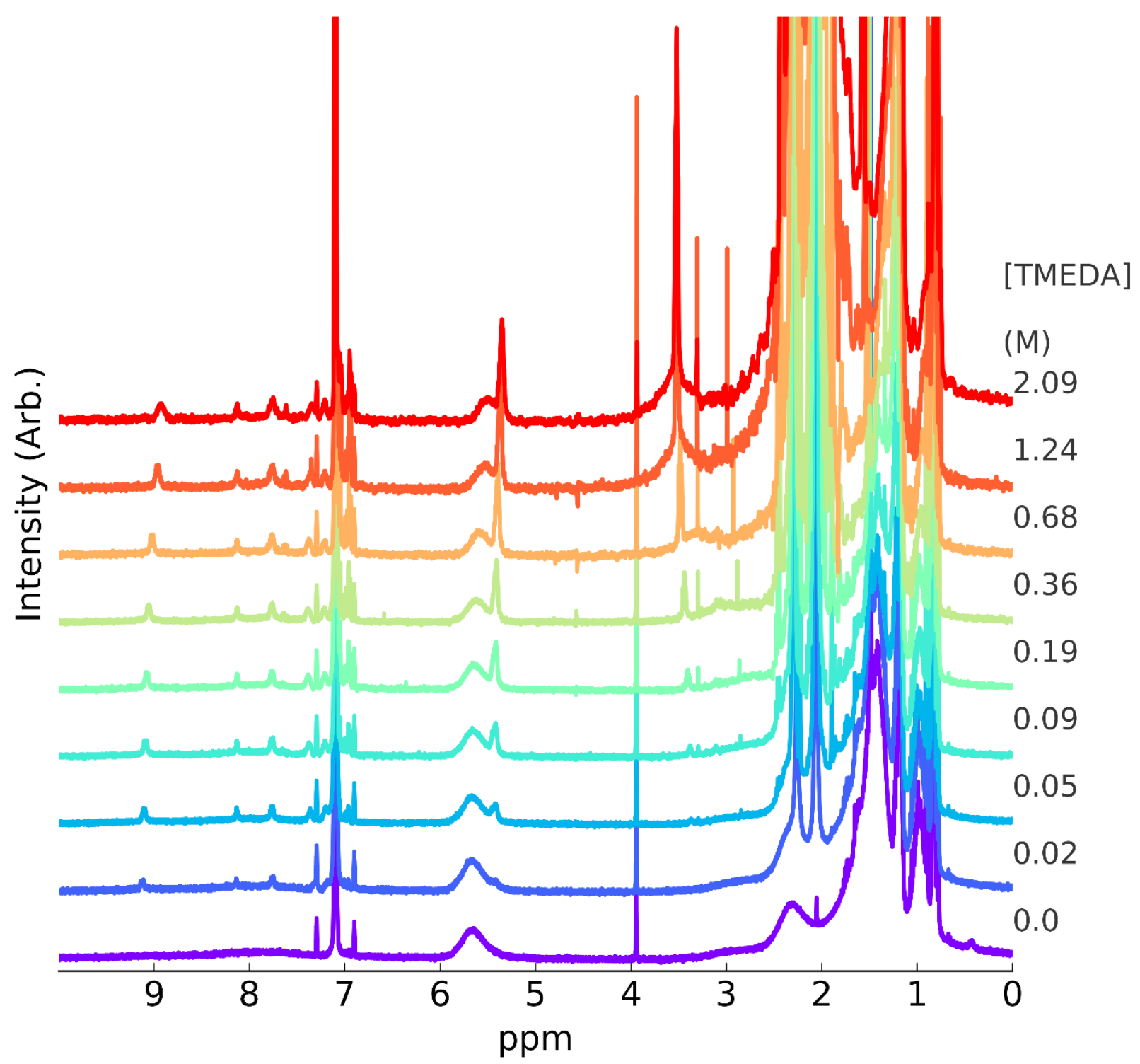

Figure S13: Complete ${ }^{1} \mathrm{H}$ NMR spectrum throughout a TMEDA titration corresponding to the suite of experiments shown in figure S12. 
9-ACA/OANCS

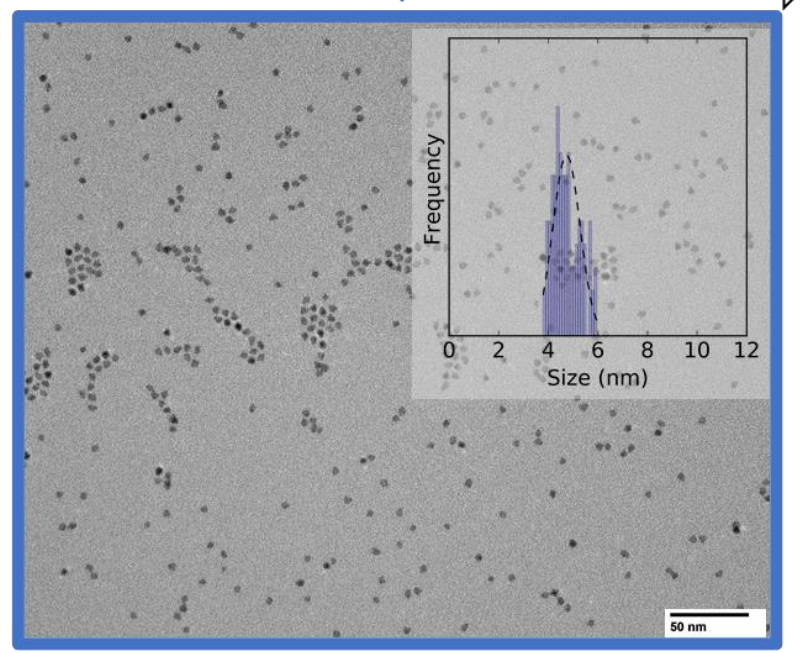

\section{After TMEDA exposure and purification}

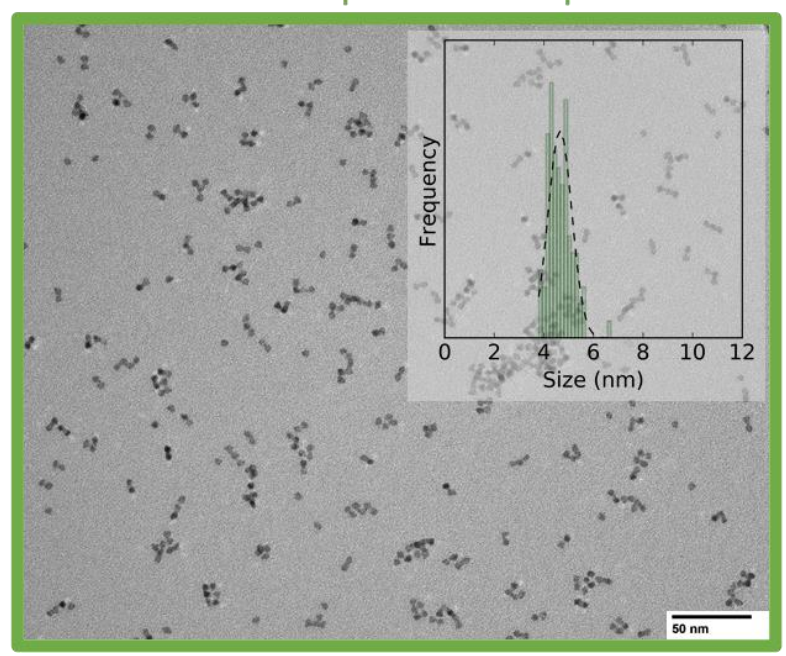

Figure S14: TEM images of a typical sample of mixed, 9-ACA/OA-passivated PbS NCs before and after being exposed to TMEDA, and then purified. Sizing histograms are inset, and show that the average size before titration is $4.7 \pm 0.5 \mathrm{~nm}$, and the average size after titration is $4.6 \pm 0.5 \mathrm{~nm}$. Other than slight changes in NC-NC packing and a reduction in the inter-NC distance following exchange, both consistent with the ligand removal, we do not observe significant structural differences following the addition of TMEDA.

\section{Comment on the vinylic proton linewidths during L-promoted Z-type ligand displacement}

(See panel D in figures S8-12). Intriguingly, for OA-only NCs (figure S8), we note a slight, initial broadening of the linewidth followed by subsequent narrowing. This rise is consistent with the initial removal of the most-reactive $\mathrm{OA}$ ligands. The subsequent decay would match the additional conformation freedom realized as coverage on facets becomes sparse. A similar rise and fall is also observed with 8\% 9-ACA coverage NCs (figure S9). By contrast, for the highest-attained 9-ACA coverages (14-16\%, figures S10-12) the linewidth is only observed to decay. This is consistent with the full removal of the most-dynamic OA a priori-during the initial $\mathrm{X}$-for-X exchange to form the mixed shell-so that the dominant effect on the linewidth from the subsequent L-type promoted Z-type ligand displacement is narrowing only, via the increased conformational freedom of the average remaining ligand due to the sparser coverage. However, a complete interpretation would require a full disambiguation of the direct impact of TMEDA on the linewidth. 


\section{Supplemental Section 3: Estimation of the concentration of bound 9-ACA.}

To provide a test of our estimate using optical absorption that only 7\% of the initial 9-ACA on mixed 9ACA/OA NCs remained bound following TMEDA titration (Figure S17), we attempted to relate the percentage of free 9-ACA observed by NMR to the number of ligands observed by absorption measurements, after having noting that the free 9-ACA integration plateaus (Figure S9-12). To test this estimate, we plot the percentage of 9-ACA observed by NMR at the end of the titration versus the number of ligands per NC observed by absorption measurements. We observe a linear correlation between the NMR and absorption experiments. This supports our assumption of near-complete removal, because if removal was substantially incomplete, a linear trend here would require the coincidence that the same non-unity percentage of ligands was removed in each experiment-in the face of the known heterogeneity in the binding.

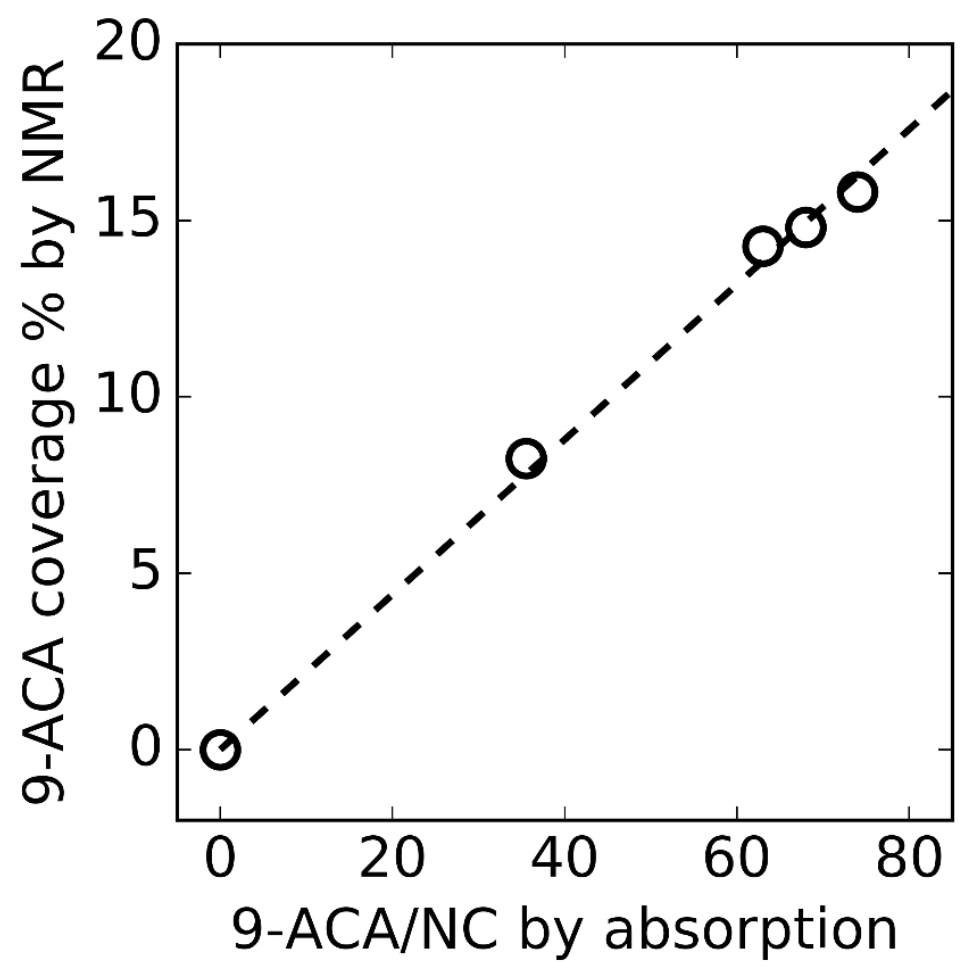

Figure S15: Comparison of the ligand coverage percentage by 9-ACA as determined by NMR and the number of 9-ACA per NC as determined by optical absorption and NMR. By measuring the absorption of solutions in which no free 9-ACA is detected, we obtain the number of bound 9-ACA per NCs. Then, using NMR, we calculate the integration of the free 9-ACA feature at the endpoint of TMEDA titration relative to the integrations of all observable ligands (free 9-ACA+ free OA+ bound OA). Assuming full displacement, this gives an independent estimate of the fractional 9-ACA coverage. We observe a clear, linear relation between these quantities, which is consistent with the assumption of near-full displacement as has been observed by NMR and absorption. See discussion above. 

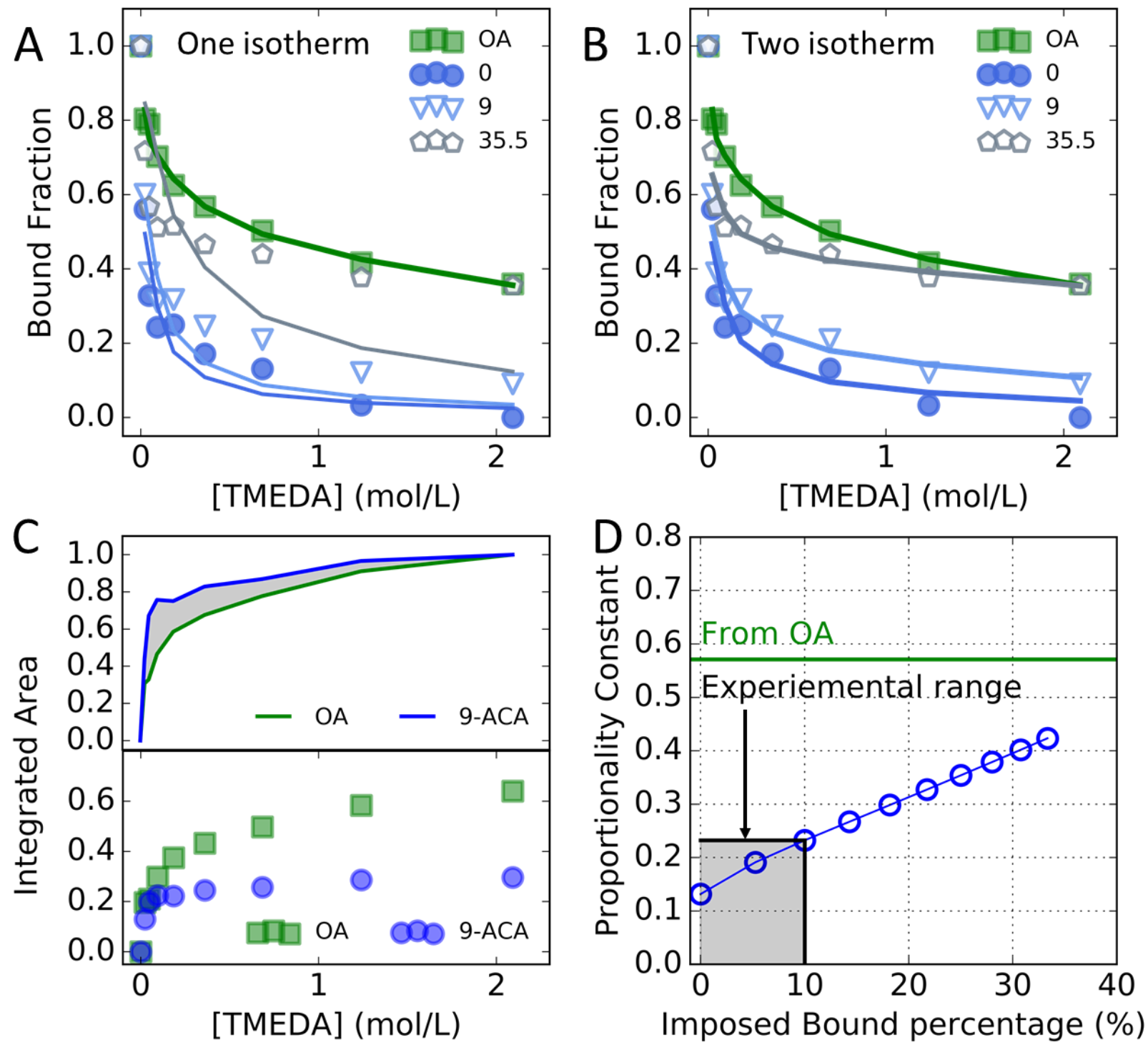

Figure S16: Sensitivity analysis of our claim that the behavior of 9-ACA in TMEDA titrations is distinct from that of OA. While our optical absorption measurements suggest a near-complete removal of 9-ACA $(<10 \%$ remaining, figure S17), we cannot directly quantify this value using ${ }^{1} \mathrm{H}$ NMR due to the difficulty in observing the bound 9-ACA resonance. Thus, we test the case where our estimate of near-complete displacement from the UV-Vis data is erroneous.

A) Measured data on L-type promoted displacement from mixed 9-ACA/OA NCs (solid symbols as indicated) as well as the preferred two-isotherm fit to the OA displacement and one-isotherm fit to the 9ACA displacement, all reproduced from Figure 3. We then generate two artificial data sets that include imagined populations of tightly bound ligands. One (hollow pentagons) is scaled to match the final free/bound ratio of OA in mixed 9-ACA/OA NCs, and the second (inverted triangles) is our estimated upper limit on the residual bound-9-ACA population given the shape of the feature in dedicated measurements (Figure S5, S19, S20) and the signal-to-noise achievable in our routine NMR. Here, either addition causes a one-isotherm fit (light blue \& grey, respectively) to poorly capture the artificial data. 
B) The same measured and artificial data from A), but showing two-isotherm fits for both artificial data sets. Here, the contributions from the two isotherms are weighted by a proportionality constant, $\alpha$ (equation $\mathrm{S} 1$, where $K_{\uparrow}$ is the equilibrium constant of the more-dynamic equilibrium, and $K_{\downarrow}$ is the constant of the more tightly-bound equilibrium, and all other variables are the same as in Equations 1-3 in the main text.). Importantly, even when introducing a large (35.5\%), experimentally unlikely residual coverage of bound 9-ACA (grey pentagons), the artificial data still shows a considerably sharper transition to the tightly bound isotherm compared to the actual OA displacement isotherm (green squares). In short, even in this extreme case, the curve-shapes remain qualitatively different, reflecting that after the initial L-tyle promoted release of 9-ACA, very little further displacement is observed. In other words, any imagined residual (undetected) population of bound 9-ACA would need to be more strongly bound than any other ligand in our experiments.

C) This distinction is also very apparent in the raw integrations (bottom panel, top panel is the same data normalized to the final point), where the integration of 9-ACA abruptly saturates while OA slowly varies.

D) Further, if we track the proportionality constant $(\alpha)$ for every imposed bound coverage, we notice that even at $35.5 \%$ it is below 0.5 , which is still less than the actual observations from the two-isotherm model of the OA displacement. By contrast, at our estimated upper limit (10\%) of the remaining bound coverage from instrument sensitivity (black squares), the fits would suggest that $\alpha<0.25$, meaning that at least $75 \%$ of 9-ACA ligands are described by the more-reactive isotherm. Therefore, our conclusion that the majority of 9-ACA is associated with the most reactive binding sites is robust to our assumptions, as it arises directly from the fitted equilibrium at low TMEDA concentrations, where any small amount of residual bound molecules have little impact. Taken together, the displacement isotherms between 9-ACA and OA are markedly different and 9-ACA is more rapidly displaced, independently of the estimated bound coverage.

$$
\theta=(1-\alpha) \frac{\left[\mathrm{L}-\mathrm{PbX}_{2}\right]}{K_{\uparrow}[\mathrm{L}]^{\mathrm{n}}+\left[\mathrm{L}-\mathrm{PbX}_{2}\right]}+\alpha \frac{\left[\mathrm{L}-\mathrm{PbX}_{2}\right]}{K_{\downarrow}[\mathrm{L}]^{\mathrm{n}}+\left[\mathrm{L}-\mathrm{PbX}_{2}\right]}
$$




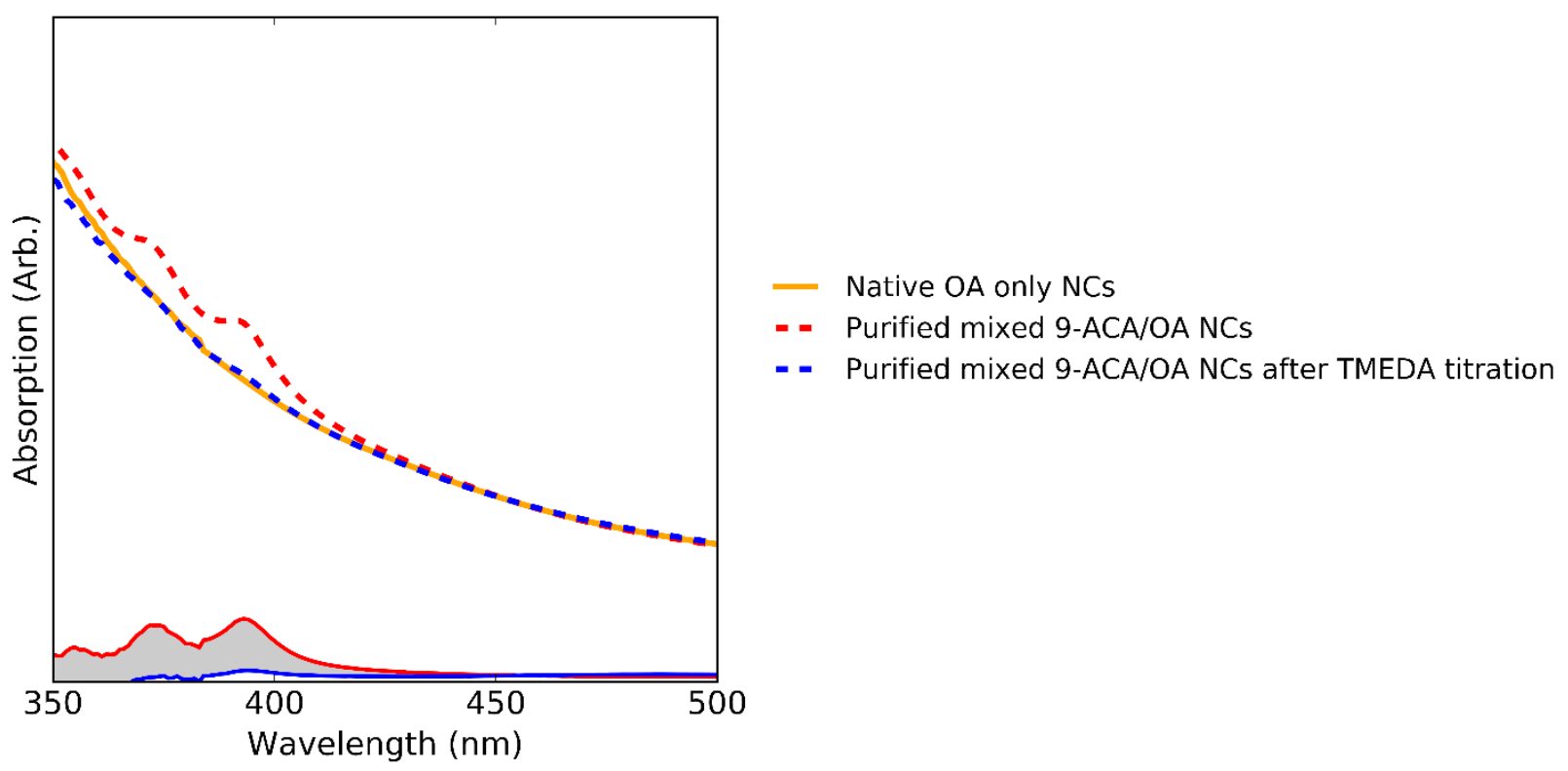

Figure S17: Example of optical absorption spectra of PbS NCs with optically-inert native OA ligands, after 9-ACA exchange (and purification), and then again after TMEDA addition and purification. Following 9ACA addition, we see the clear signature of 9-ACA (red curve) a top that of the native NC (green curve), indicating a mixed 9-ACA/OA ligand shell. We consider that the concentration of free 9-ACA ligands is low in these samples following purification, because signatures of un-bound 9-ACA are absent in ${ }^{1} \mathrm{H} N M R$ measurements on comparable samples. (Figures S9-S12) After TMEDA addition and subsequent purification with acetone (blue curve) the 9-ACA signature is significantly reduced. Subtracting the signal from the native NC from that of the 9-ACA exchanged/TMEDA exposed curve allows the contributions from residual 9-ACA (presumably bound) to be extracted (dashed lines). We then estimate that approximately $7 \%$ of the 9-ACA remains on the NC after the TMEDA titration. 


\section{Supplemental Section 4: Data fitting and extra characterization of the exchanged NCs}

In the main manuscript we employed the fitting procedure as described by Drijver et al. ${ }^{11}$ However alternate forms of this equation have been developed to demonstrate the binding site heterogeneity. In this section, we alternatively fit our data using the form of the displacement isotherm equations derived by Saniepay and Beaulac ${ }^{12}$, and used by Kessler and Dempsey on PbS NCs (Equations 1-4). ${ }^{13}$ These equations have the elegant consequence that a one-isotherm process appears as a straight line (Equation 1), allowing simple visual confirmation of binding-site heterogeneity if any curvature is observed.

$$
\begin{gathered}
\frac{[\mathrm{NC}]}{\left[\mathrm{B}-\mathrm{PbX}_{2}\right]}=\frac{1}{N}+\frac{K \varphi}{N}(1) \\
\frac{[\mathrm{NC}]}{\left[\mathrm{B}-\mathrm{PbX}_{2}\right]}=\frac{\varphi^{2} K_{1} K_{2}+\left(K_{1}+K_{2}\right)+1}{\varphi\left(N_{1} K_{2}+N_{2} K_{1}\right)+N_{t o t}} \\
\varphi=\frac{[\mathrm{L}]}{\left[\mathrm{L}-\mathrm{PbX}_{2}\right]}(3) \\
N_{\text {tot }}=N_{1}+N_{2}(4)
\end{gathered}
$$

Here, $[\mathrm{NC}]$ is the NC concentration, $\varphi$ is ratio of the concentrations of free and coordinated L-type ligands, and other quantities are the same as in the main text: $\left[\mathrm{B}-\mathrm{PbX}_{2}\right]$ is the concentration of bound Z-type ligands, $N_{i}$ is the amount of Z-type ligand binding sites per NC for each simultaneous binding equilibrium considered, $K_{i}$ is the equilibrium constant for a given displacement isotherm, $[\mathrm{L}]$ is the L-type ligand concentration (in this case TMEDA), and $\left[\mathrm{L}-\mathrm{PbX}_{2}\right]$ is the concentration of free Z-type ligands. Comparing to our data, we again observe that our unexchanged NCs fit well the two-isotherm model by Kessler (equation 2)

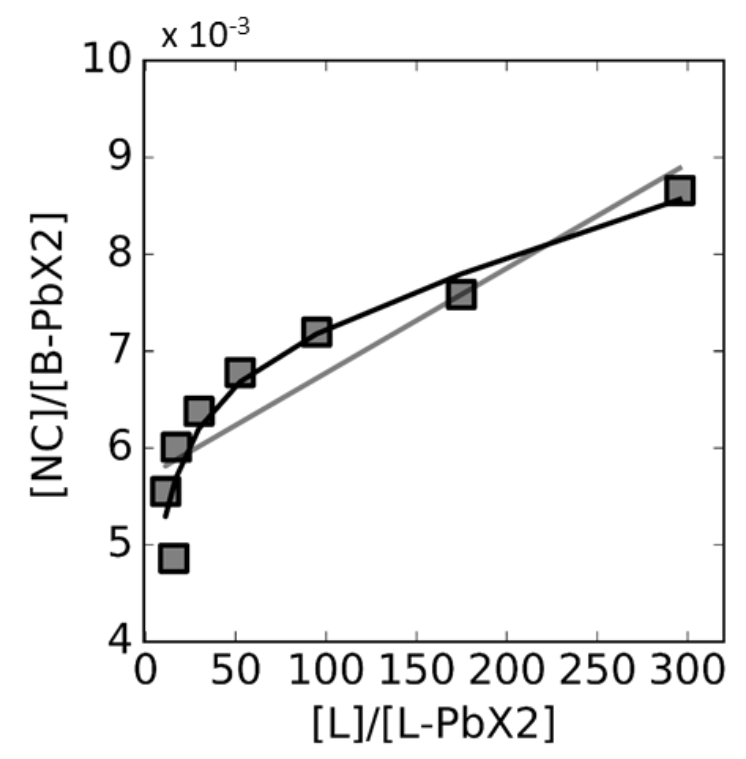

Figure S18: Displacement isotherms for OA-only NCs studied with the methodology by Kessler et al. These data are the same as shown in Figure S8. The grey line is a fit using a one-isotherm model, while the black curve represents a two-isotherm fit. The good fit with a two-isotherm model further confirms the surface binding heterogeneity of the NCs used in this study. 

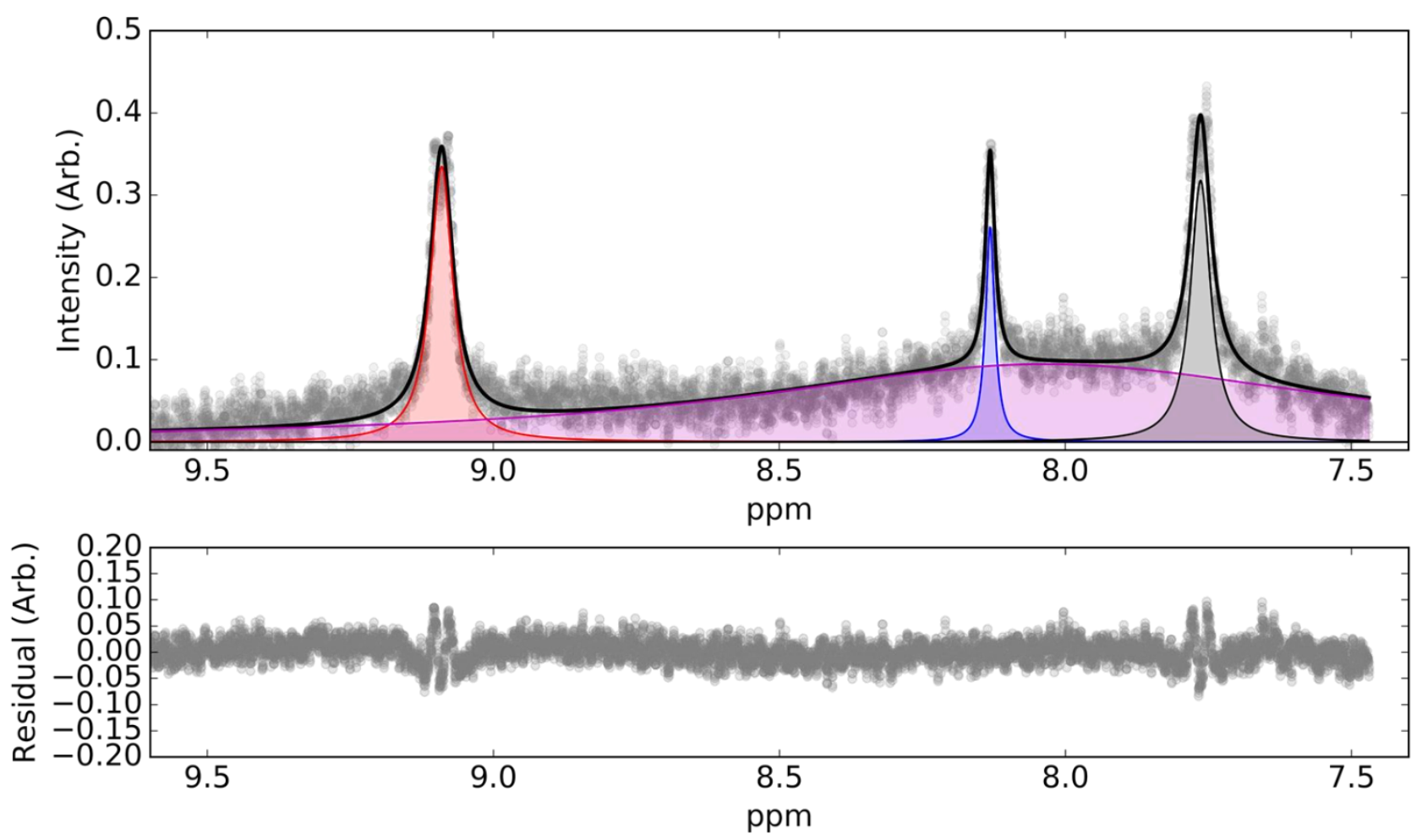

Figure S19: Example of 9-ACA region fit using three Lorentzians to capture the free 9-ACA resonances (red blue and gray curves) and a fourth Lorentzian to capture the background (purple). This method shows that the rations between the integrations of three peaks of free 9-ACA peaks remain consistent (Figure S22). However, we note that these integrations differ slightly the 2:1:2 expected ratio. We ascribe this to the difficulty in fitting the background and fully account for its contribution integration relative to the other peaks due to its breath and the influence of other (broadened) features centered at smaller chemical shifts (c.f. Figure S5, S34). 

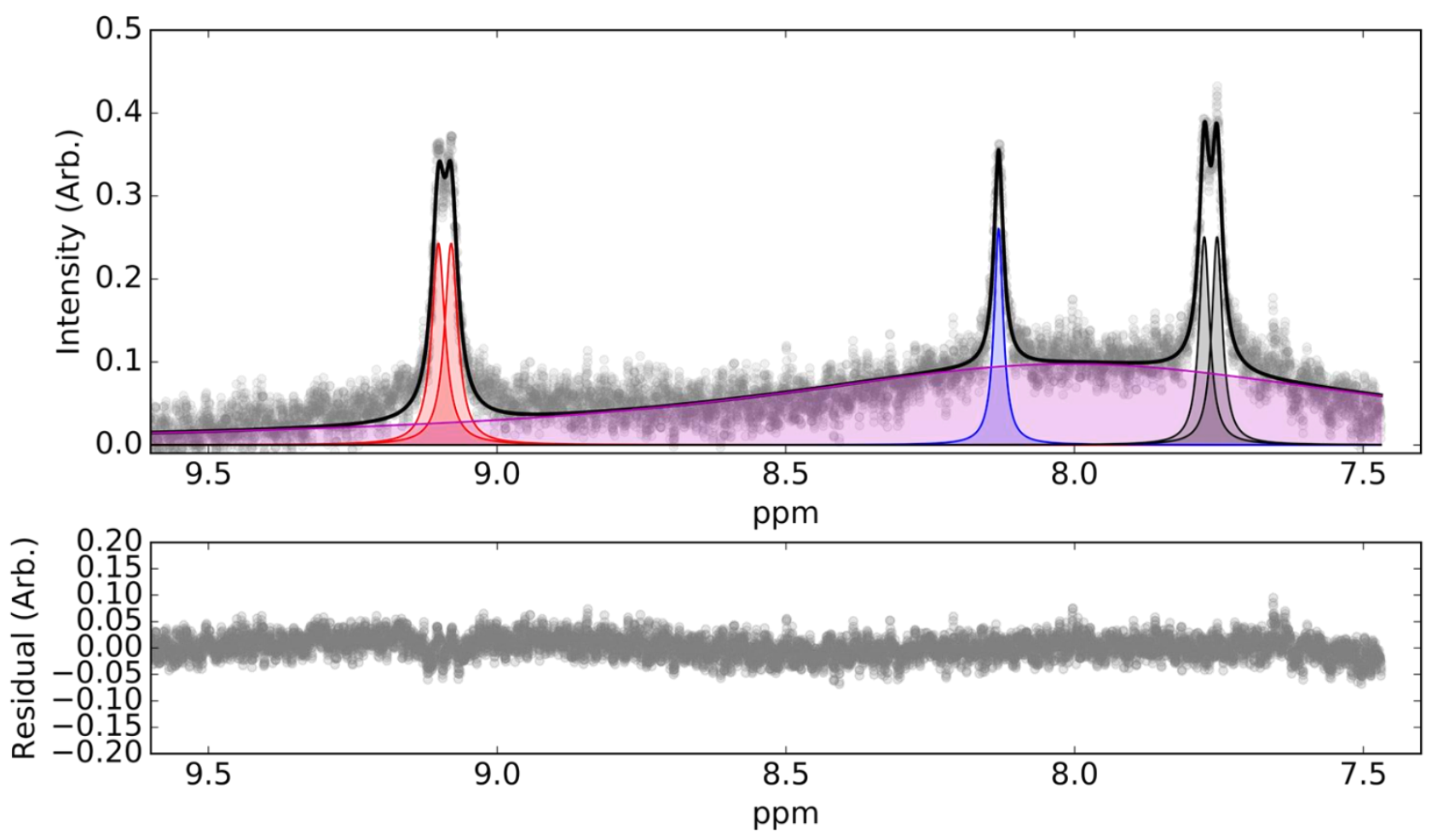

Figure S20: Same example of 9-ACA region as figure S19. This time we fitted the peaks around 9.1 and 7.7 ppm as doublets using two identical Lorentizians with different center positions. While this method better-captures the profile of the resonance, the final integrations are not significantly different from the one-peak fits in Figure S19 (c.f. Figure S21), so we employed the simpler fitting procedure to generate our data.
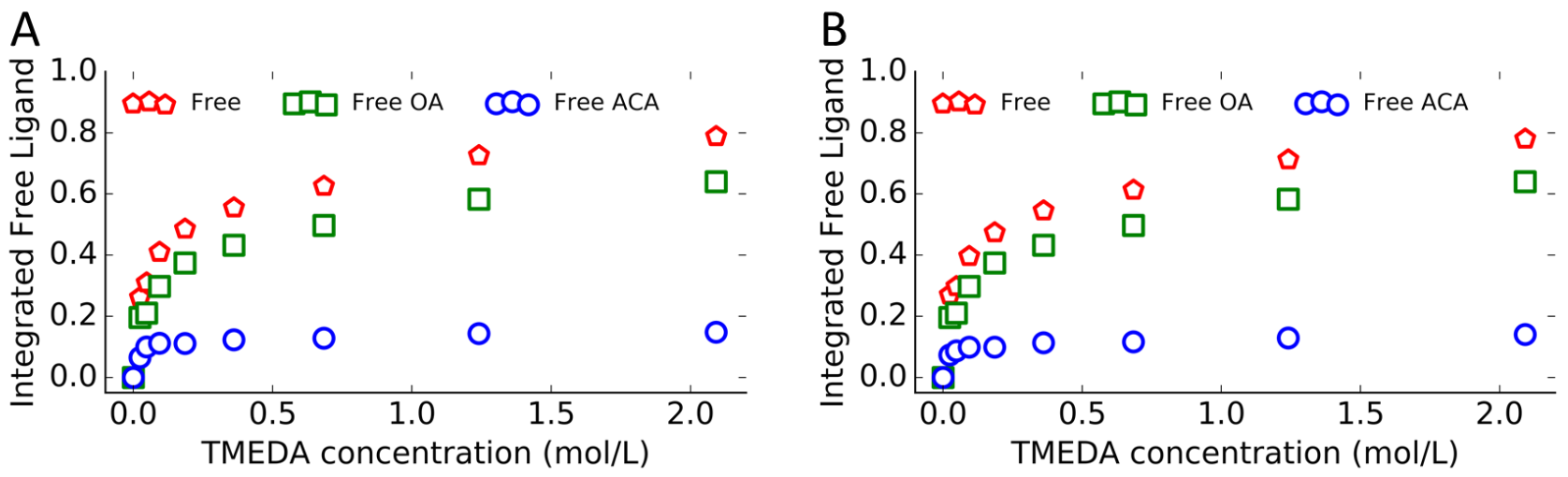

Figure S21: Observed integrations for the two different methods. A) modelling every peak as a singlet, and B) modelling the peaks around 9.1 and $7.7 \mathrm{ppm}$ as doublets. We observe no significant difference in the extracted data, thus we employed the simpler fitting method. 


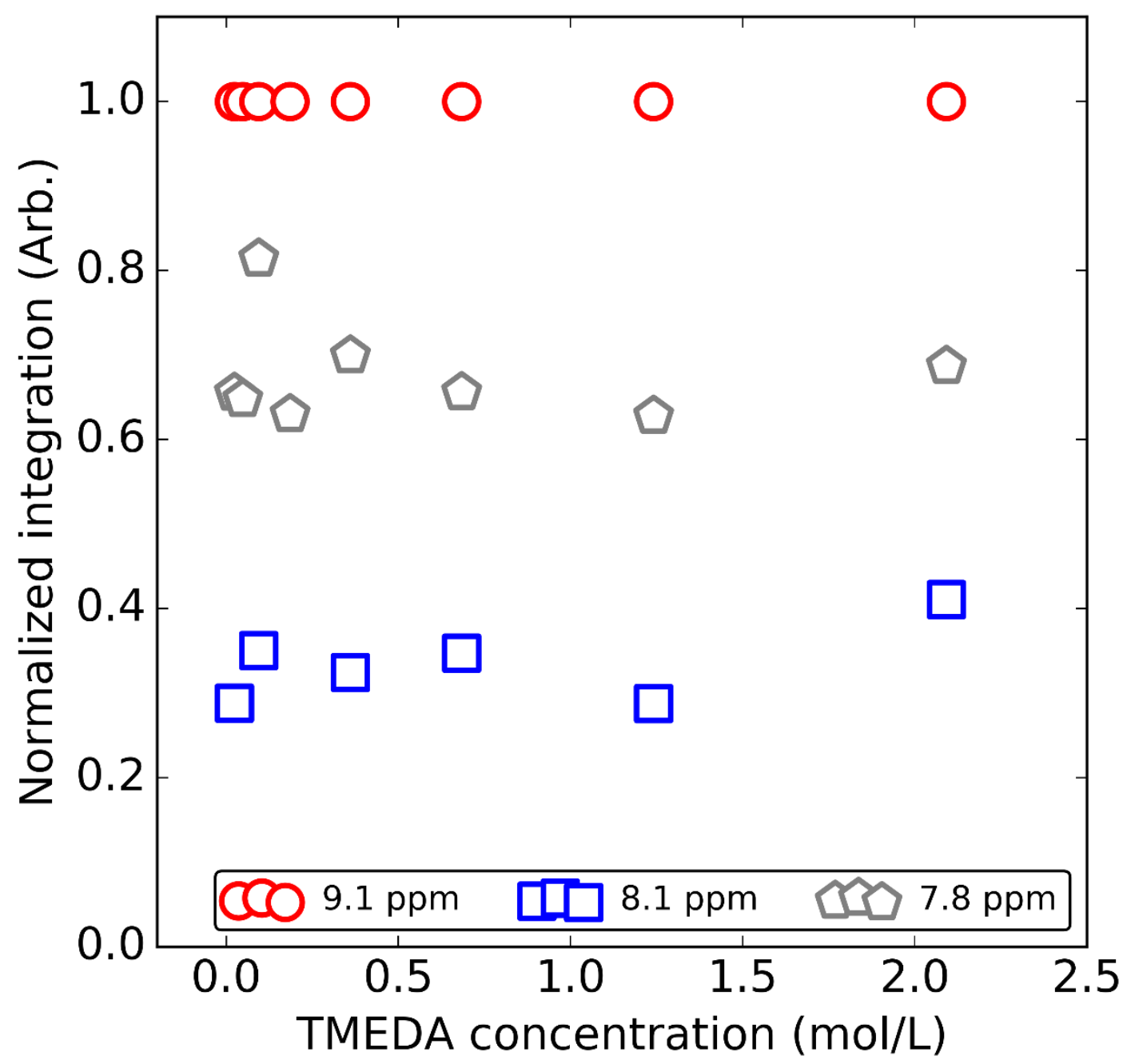

Figure S22: Relative integrations as compared to the peak around $9.1 \mathrm{ppm}$ of the three-proton resonances of free 9-ACA obtained through the fitting procedure in Figure S19. We observe that the ratios remain constant throughout the titration, validating our use of the proton resonance at $9.1 \mathrm{ppm}$ for our displacement isotherm analysis. The ratios vary somewhat from expectations (see above), though we ascribe this to difficulties in modelling the background, which more-strongly contributes to the measured integrations of the peaks at 8.1 and $7.8 \mathrm{ppm}$, both of which appear to be underestimated here. 


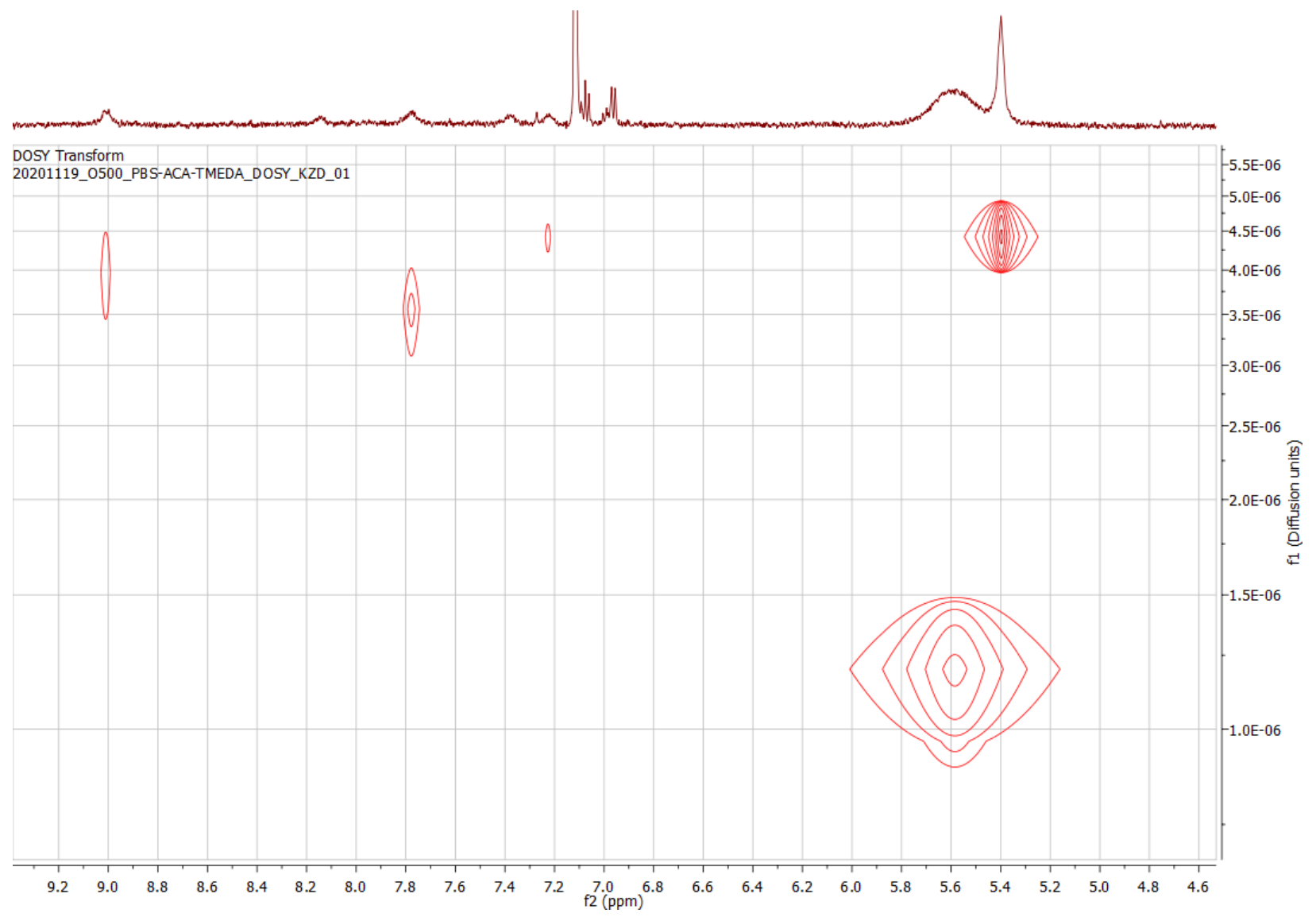

Figure S23: Diffusion ordered spectroscopy (DOSY) of mixed 9-ACA/OA PbS NCs that have undergone a partial Z-type ligand displacement with TMEDA. We observe two families of peaks: narrow ones with large diffusion constants, and broad ones with smaller diffusion constants. The broad peak is due to bound OA (5.65 ppm), no bound 9-ACA is observed as previously mentioned (Figure S5, S19, S20). The fast-diffusing species are assigned to free OA $(5.5 \mathrm{ppm})$ and free 9-ACA (9.1 ppm and $7.8 \mathrm{ppm})$. We thus confirm that the new peaks appearing during TMEDA titrations are due to free OA and 9-ACA species. 


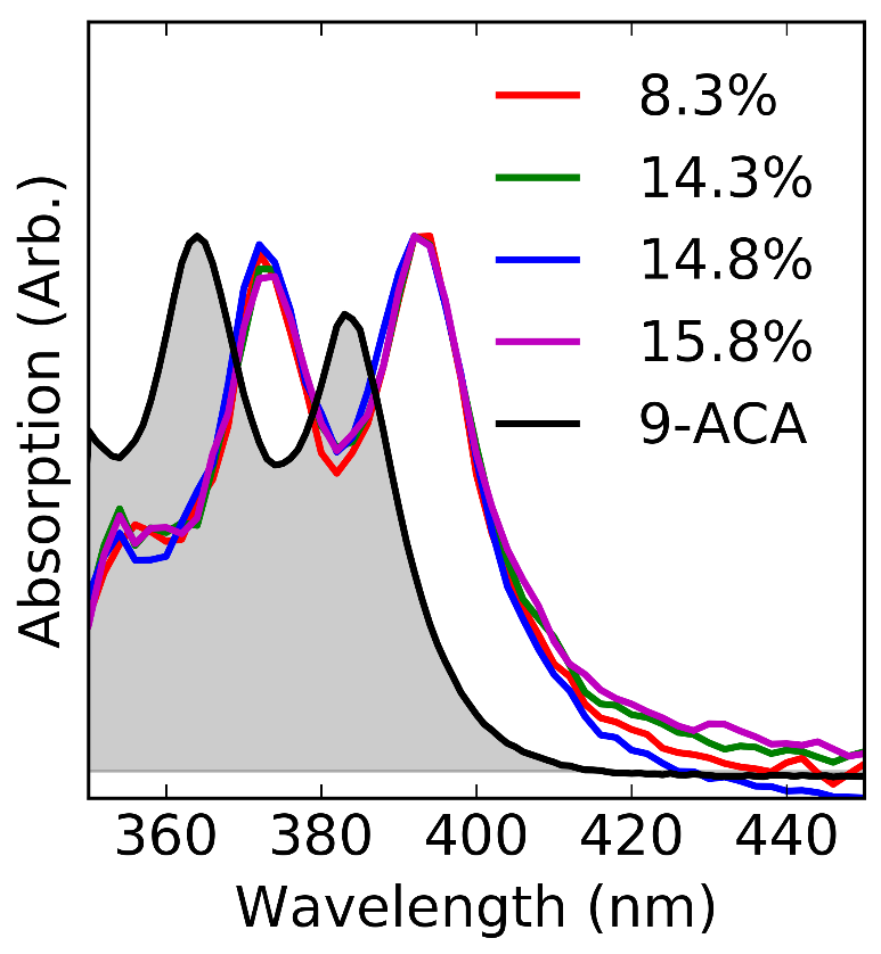

Figure S24: Absorption spectrum of free 9-ACA in solution (black line with solid grey fill) and the extracted absorption spectra of 9-ACA bound to the surface of PbS NCs (coloured lines). (See Methods for sample preparation including ligand exchange and purification.) The absorption of bound molecules is obtained from the absorbance of the hybrid particles in solution by subtracting the measured optical absorption of a solution of NCs from the same batch, prepared to a similar concentration, and then normalized at $\lambda=300$ $\mathrm{nm}$ (a wavelength where 9-ACA has little absorption.) Finally, the 9-ACA spectra are normalized to the absorption peak at $\lambda=390 \mathrm{~nm}$. Raw spectra are presented above as subpanel $E$ in Figures S9-12.

The reproducibility of the shape of the extracted spectra across samples is consistent with effective isolation of the contribution from 9-ACA. We note that the absorption of surface-bound 9-ACA is redshifted by $\sim 10 \mathrm{~nm}$, which may reflect dipolar coupling between aggregated ligands ${ }^{14}$ and/or weak coupling to the adjacent nanocrystal. ${ }^{15}$ We also observe a slight decrease in the strength of the 0-1 sideband relative to the phonon-less $0-0$ transition, consistent with reduced exciton-phonon coupling. ${ }^{14}$ 

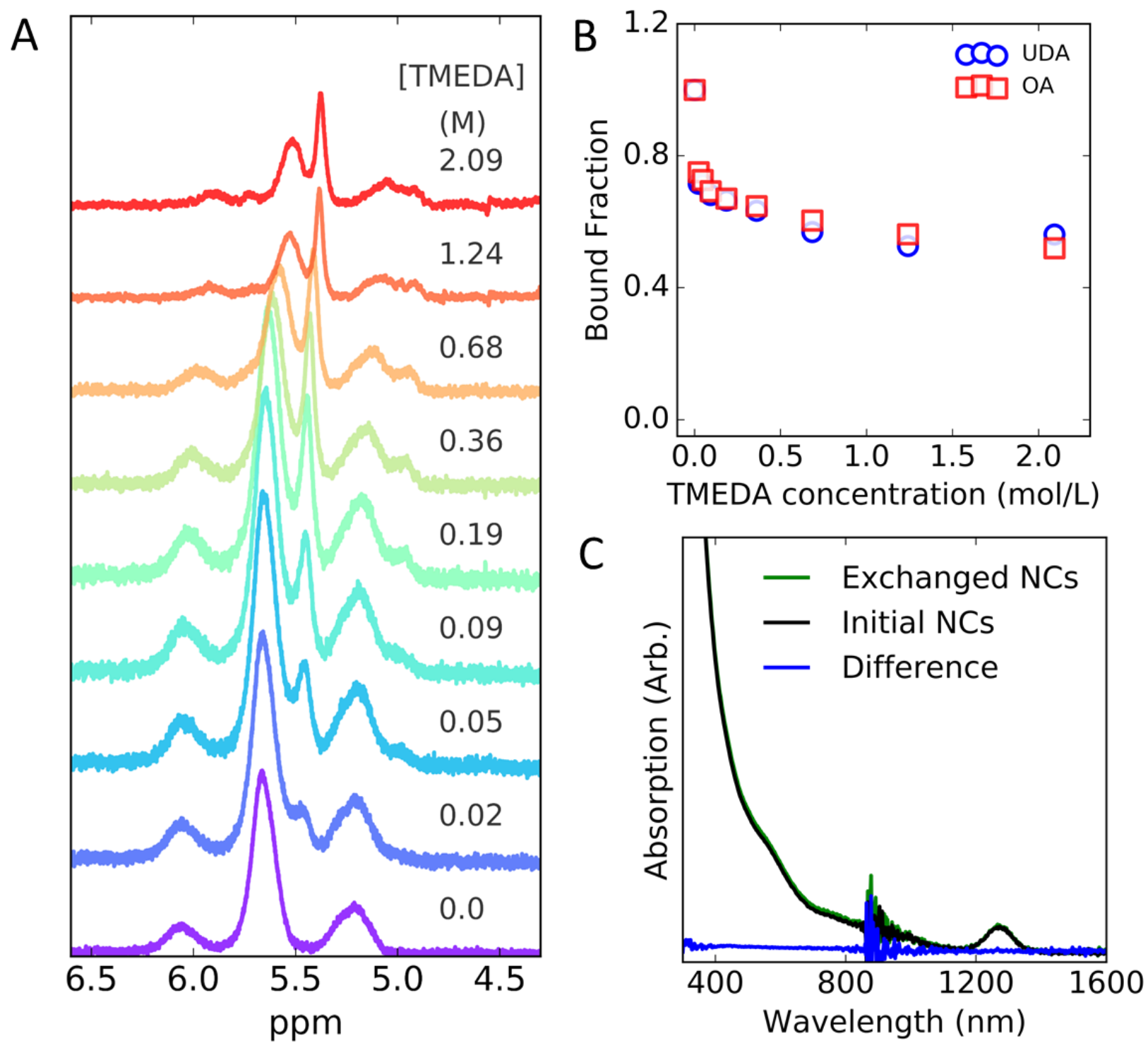

Figure S25: A) L-type promoted Z-type ligand displacement for UDA exchanged NCs, with an 32\% UDA coverage as determined by the respective integrated peaks from UDA and OA.

A) ${ }^{1} \mathrm{H}$ NMR spectra of the resonances for free and bound OA and UDA from solutions of mixed OA/UDA NCs to which increasing amounts of TMEDA are added.

B) Displacement isotherms for OA (red square) and UDA (blue circles). We observe that both isotherms are very similar suggesting equal displacement of OA and UDA throughout the TMEDA titration.

C) Absorption spectra of the exchanged NC. We observe no significant difference between the absorption of the unexchanged and UDA exchanged NCs. 
Supplemental Section 6: Variable temperature ${ }^{1} \mathrm{H}$ NMR measurements:
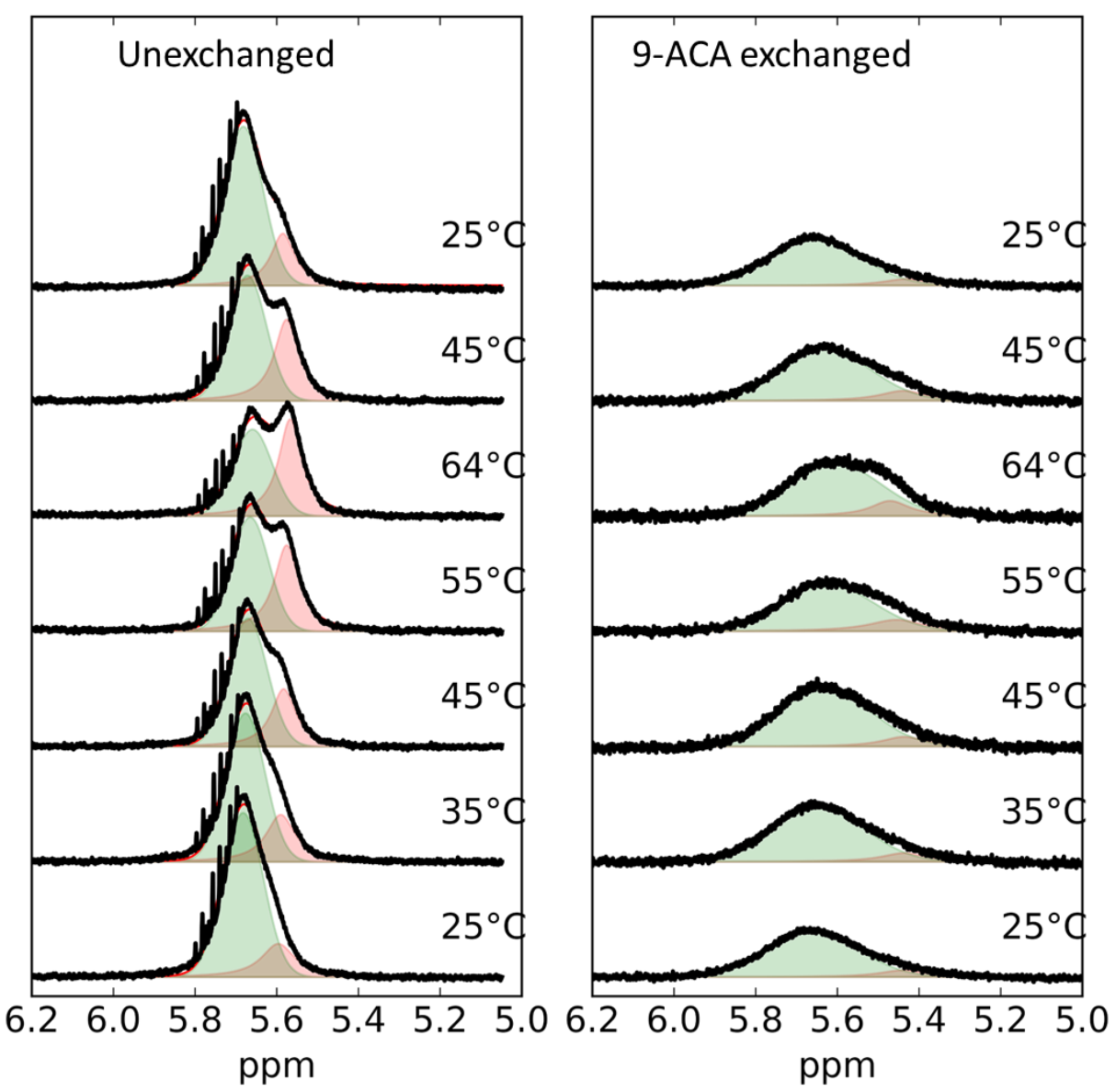

Figure S26: Evolution of the vinylic proton resonances with temperature for OA-only (left) and mixed 9ACA/OA NCs (right). Heating the samples from $25^{\circ} \mathrm{C}$ to $64^{\circ} \mathrm{C}$ and then cooling them back to $25^{\circ} \mathrm{C}$ we observe distinct behavior between the NCs solutions with the differing ligand shells. All peaks are fitted with a Gaussian for bound features (green) and Lorentzian for free features (red). ${ }^{11}$ The multiple sharp features on the left flank of the vinylic resonance for OA-only NCs are due to residual ODE from the synthesis. We note in passing the significant broadening of the vinylic OA signal when 9-ACA is present, this is discussed below in Supplemental Section 7.

As summarized in the main text (Figure 4), we observe that considerably less $O A$ is released upon heating when 9-ACA is present on the surface. We consider the release of OA is due to an X-type binding site heterogeneity where more reactive sites release OA rapidly. When 9-ACA is exchanged, these more reactive X-type $O A$ (at the facet-edges) are preferentially replaced (due to the ligand-ligand factors discussed elsewhere). This would have the consequence that only tightly bound OA remains, explaining the observed limitation of subsequent thermal displacement. In addition, we observe no 9-ACA displacement in these measurements (See Figure S27), which is consistent with the low solubility of 9ACA in benzene $d 6$ as well as the possibility of ligand aggregation via stacking. Taken together, this suggests that 9-ACA is efficiently replaces the highest reactivity OA, which are preferentially on facet edges. ${ }^{11,13}$ 


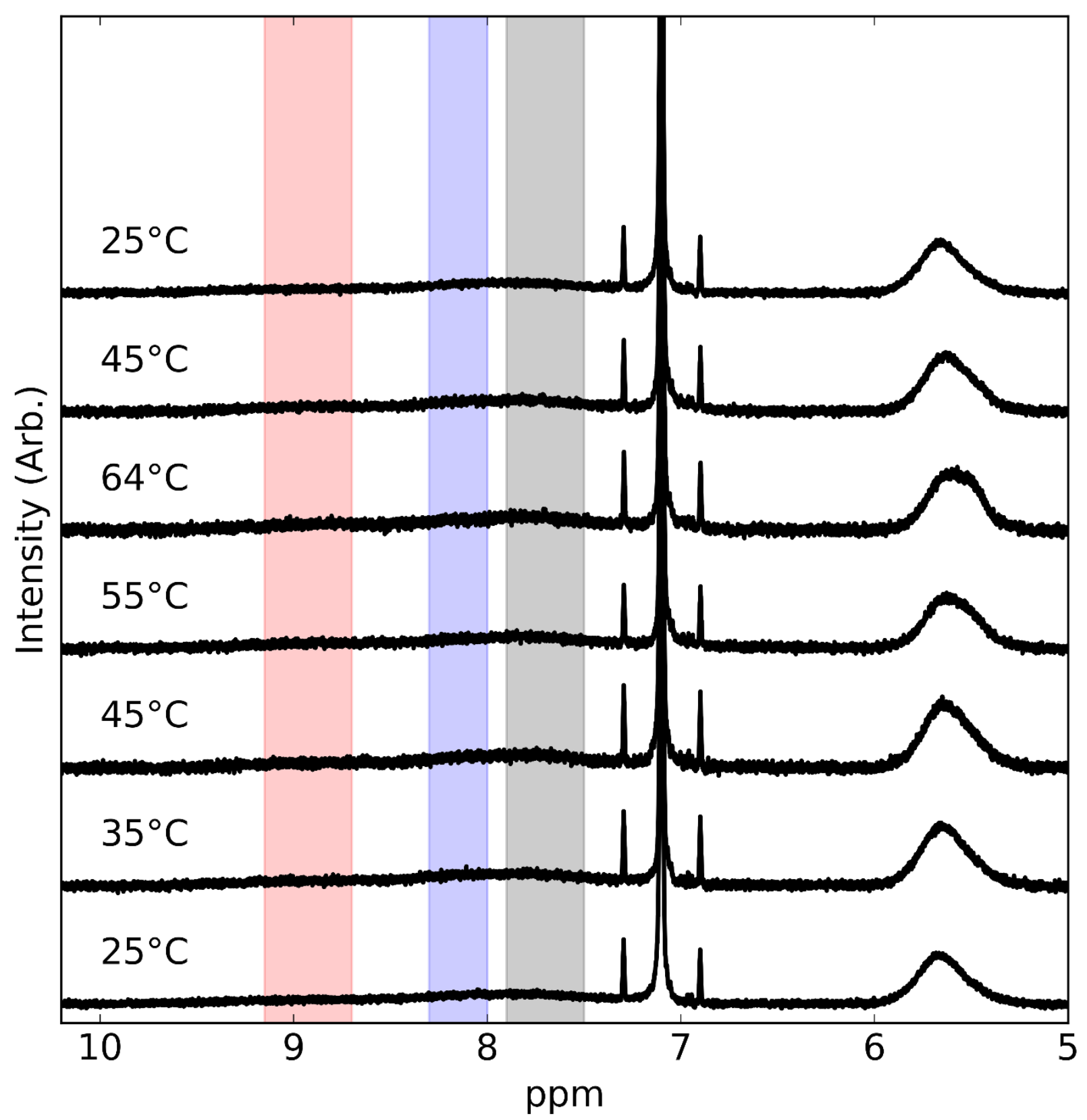

Figure S27: Temperature evolution of the ${ }^{1} \mathrm{H}$ NMR spectrum of mixed 9-ACA/OA NCs. The feature around $5.7 \mathrm{ppm}$ is associated with bound and free vinylic protons of OA and is discussed in figure S16. Between 9.5 and $7.5 \mathrm{ppm}$ are the very broad proton resonances associated with bound 9-ACA (Figure S5, S16, S17). Highlighted in red, blue, and grey are the regions where free 9-ACA features appear as per figure S16. No signal is observed to change with temperature indicating that 9-ACA is not thermally displaced. 
Here we use a well-established linewidth analysis ${ }^{16,17}$ procedure to extract homogeneous and heterogeneous contributions to the breadth of the ${ }^{1} \mathrm{H}$ resonances that we observe in $\mathrm{NMR}$, and include a brief explanation here for convenience. In the absence of magnetic field heterogeneity, the peak width can be used to approximate $T_{2}$ using the following equation:

$$
F W H M(H z)=\frac{1}{\pi T_{2}}(1)
$$

However, when there is heterogeneity, the peak width gives $T_{2}^{*}$ which is related to the homogeneous $T_{2}$ as follows:

$$
\frac{1}{T_{2}^{*}}=\frac{1}{T_{2}}+\frac{1}{T_{\text {hetero }}}
$$

Where $T_{\text {hetero }}$ is the heterogeneous contribution to the linewidth. To calculate the homogenous $T_{2}$ we used the CPMG pulse sequence. ${ }^{16,17}$ This gives an exponentially decaying signal with increased delay time, which can be fit using the following equation:

$$
M_{x y}=M_{x y}(0) e^{-t / T_{2}}
$$

Thus, using $T_{2}$ and Equation 1, we can estimate the homogeneous linewidth in isolation. Further, from the full linewidth and homogeneous linewidth, we can then use Equation 2 to estimate the heterogeneous linewidth.

The following table summarizes the results from this procedure, and the raw data is presented in the six supplementary figures that follow.

Table S1:

\begin{tabular}{cccccc}
\hline $\begin{array}{c}\text { Ligand } \\
\text { exchanged }\end{array}$ & $\begin{array}{c}\boldsymbol{T}_{\mathbf{2}} \text { by CPMG } \\
(\mathbf{m s})\end{array}$ & FWHM (Hz) & $\begin{array}{c}\text { Homogeneous } \\
\text { FWHM (Hz) }\end{array}$ & $\begin{array}{c}\text { Heterogeneous } \\
\text { FWHM (Hz) }\end{array}$ & Figure \\
\hline Unexchanged & 27.5 & 60.5 & 11.6 & 48.9 & $\mathrm{~S} 28$ \\
\hline UDA & 30.0 & 57.27 & 10.6 & 46.7 & $\mathrm{~S} 29$ \\
\hline UDA & 29.9 & 61.23 & 10.63 & 50.1 & $\mathrm{~S} 30$ \\
\hline UDA & 26.9 & 64.46 & 11.8 & 50.7 & $\mathrm{~S} 31$ \\
\hline 9-ACA & 36.4 & 110.1 & 8.7 & 101.3 & $\mathrm{~S} 32$ \\
\hline 9-ACA & 36.17 & 125.34 & 8.8 & 116.5 & $\mathrm{~S} 33$ \\
\hline
\end{tabular}



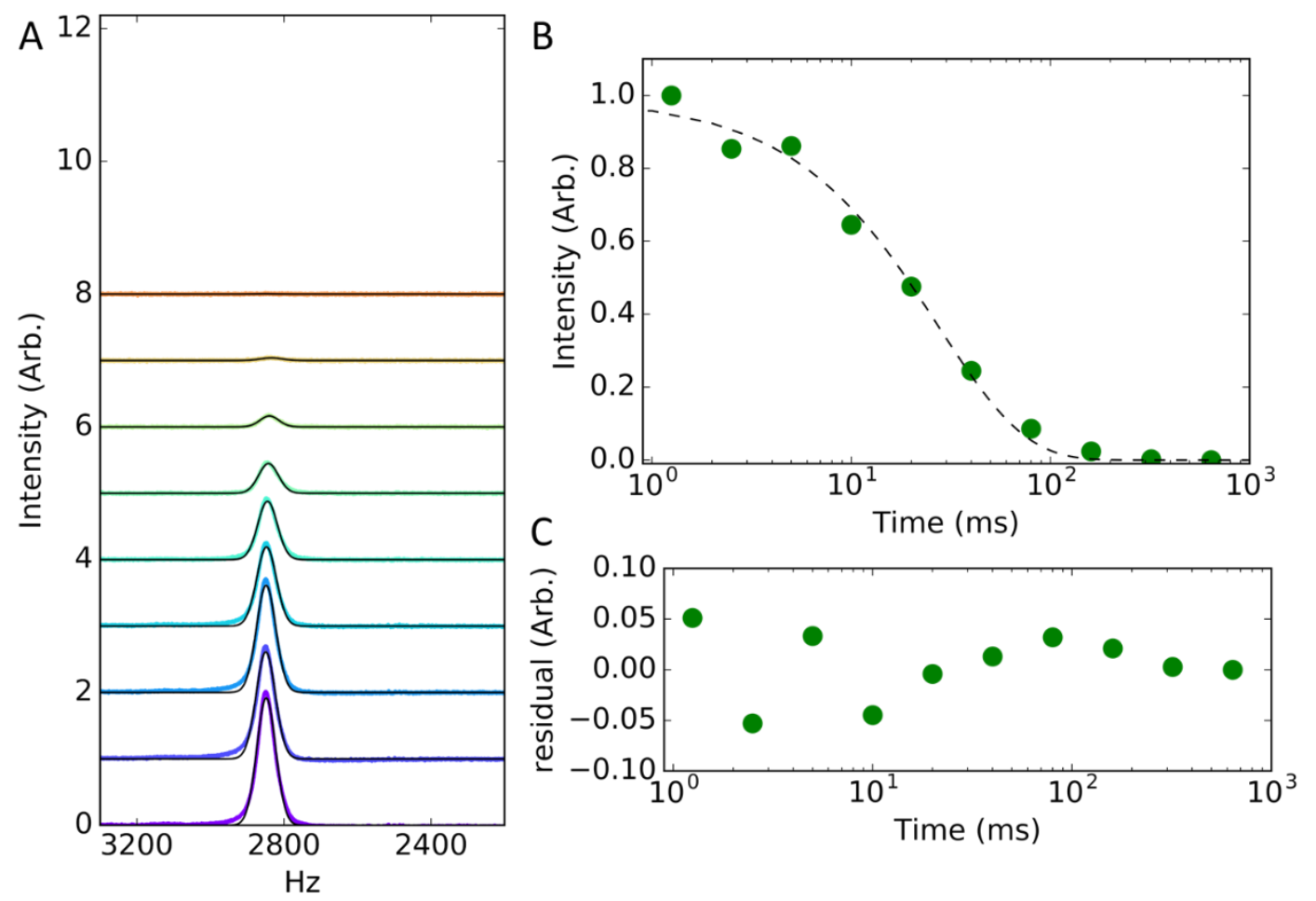

Figure S28: Linewidth analysis for unexchanged NCs. A) Signal observed at varying time delay. B) Integrated intensity decay with delay time. The data was fitted with equation 3 to yield a $T_{2}$ of $27.5 \mathrm{~ms}$. Given the peak FWHM of $60.5 \mathrm{~Hz}$ we obtain a homogenous FWHM of $11.6 \mathrm{~Hz}$ and heterogeneous FWHM of $48.9 \mathrm{~Hz}$. C) Residual to the fit in $\mathrm{B}$. 

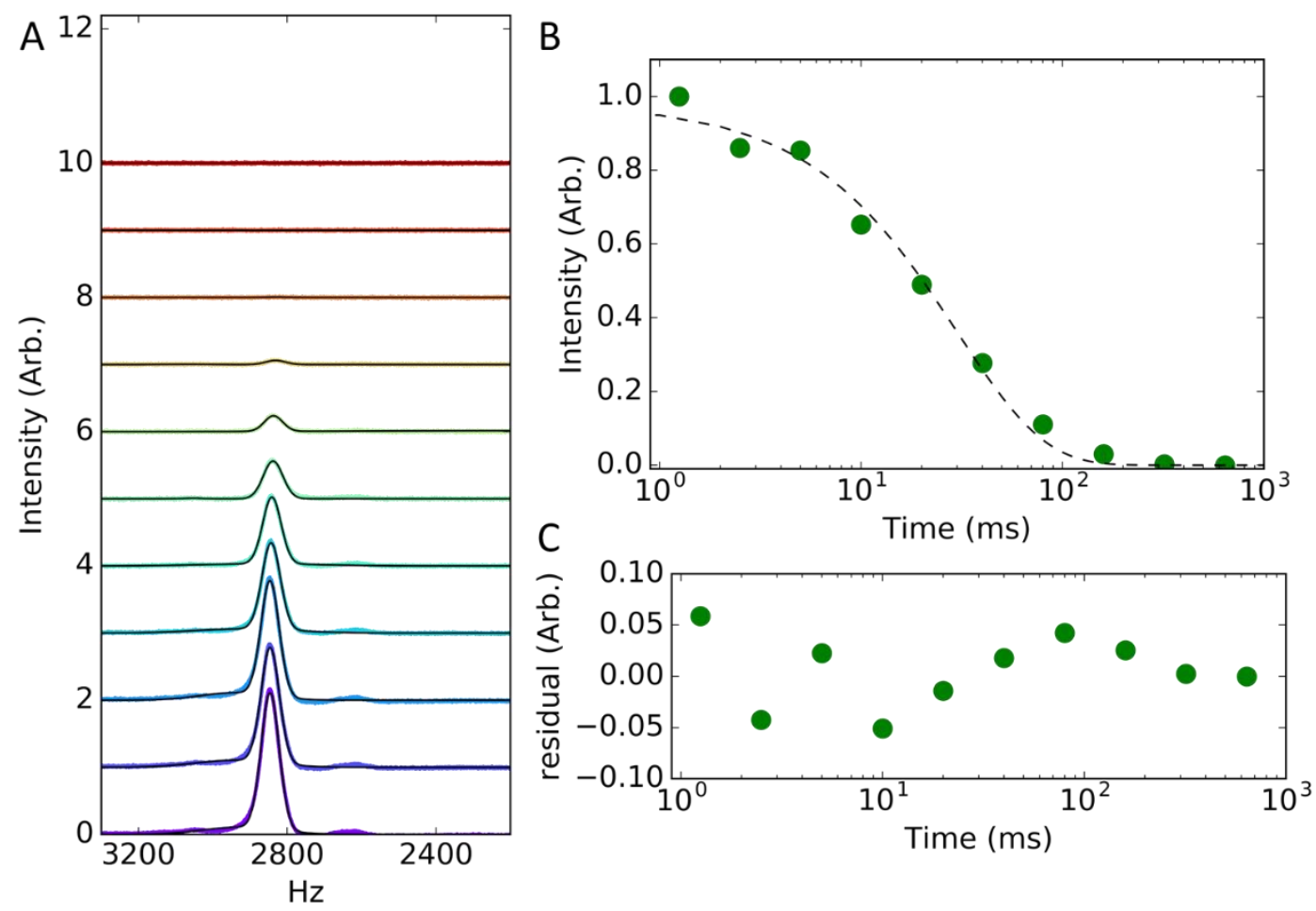

Figure S29: Linewidth analysis for UDA exchanged NCs. A) Signal observed at varying time delay. B) Integrated intensity decay with delay time. The data was fitted with equation 3 to yield a $T_{2}$ of 30.0 ms. Given the peak FWHM of $57.3 \mathrm{~Hz}$ we obtain a homogenous FWHM of $10.6 \mathrm{~Hz}$ and heterogeneous FWHM of $46.7 \mathrm{~Hz}$. C) Residual to the fit in $\mathrm{B}$. 

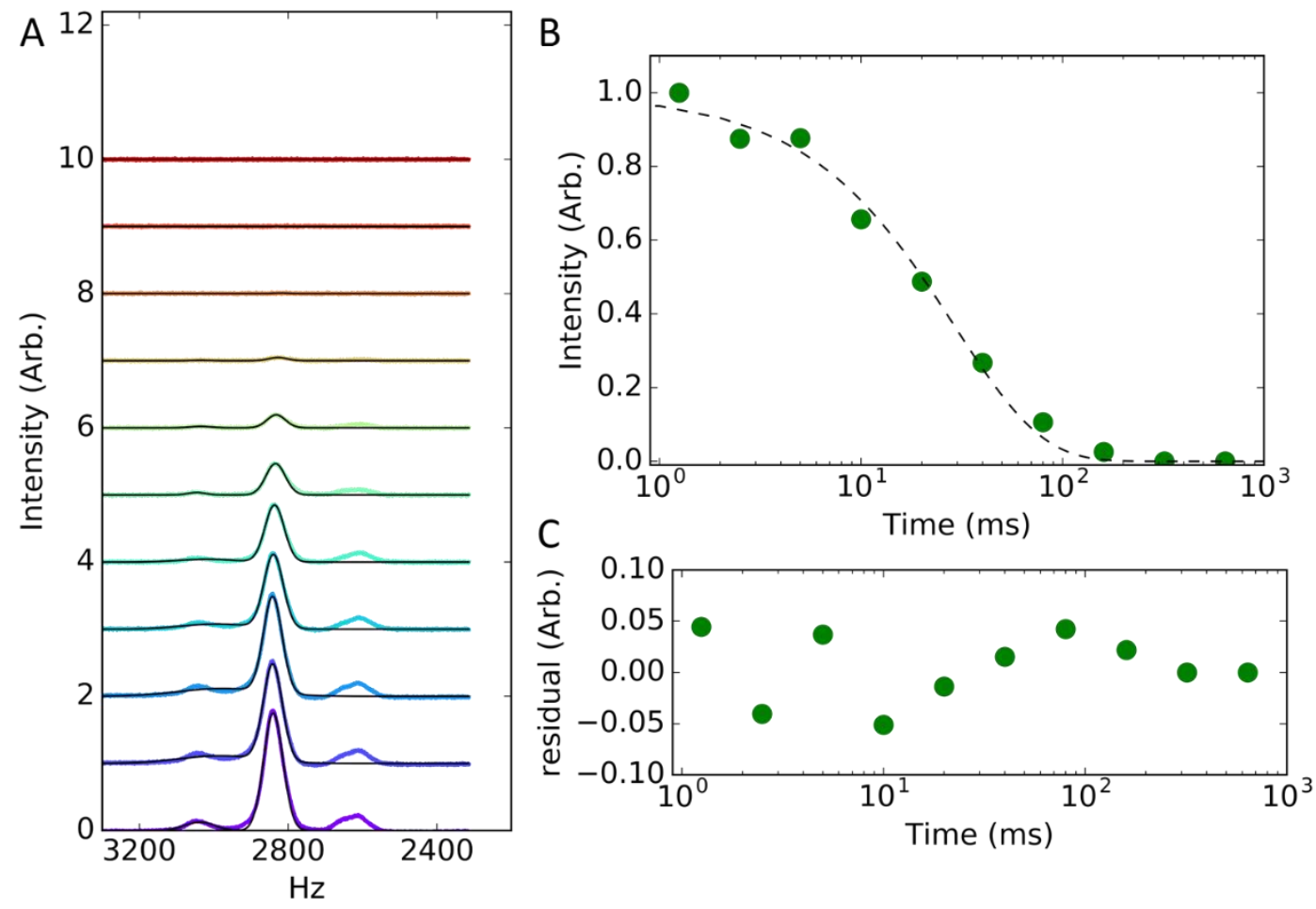

Figure S30: Linewidth analysis for UDA exchanged NCs. A) Signal observed at varying time delay. B) Integrated intensity decay with delay time. The data was fitted with equation 3 to yield a $T_{2}$ of 30.0 ms. Given the peak FWHM of $61.23 \mathrm{~Hz}$ we obtain a homogenous FWHM of $10.6 \mathrm{~Hz}$ and heterogeneous FWHM of $50.1 \mathrm{~Hz}$. C) Residuals to the fit in B. 

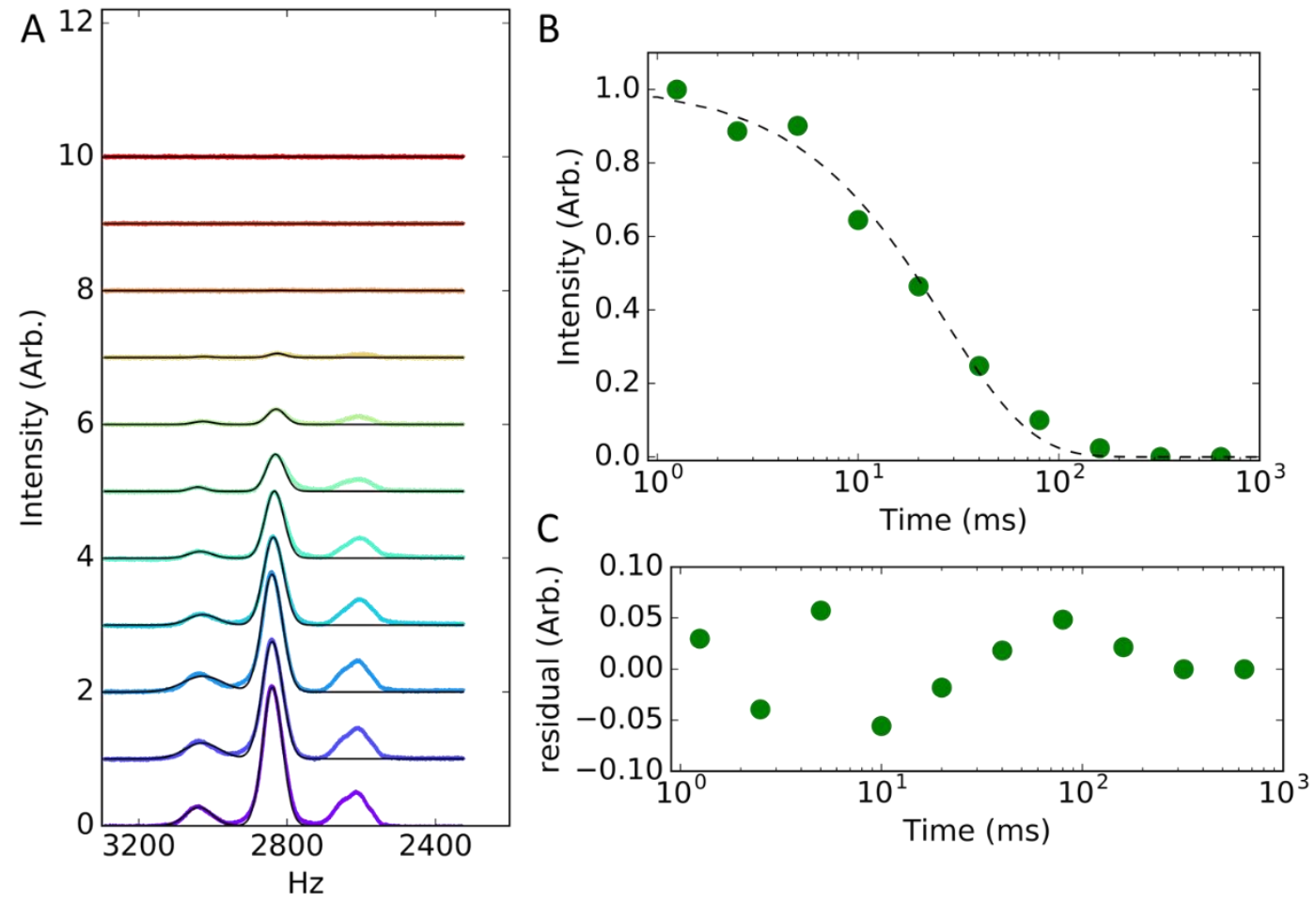

Figure S31: Linewidth analysis for UDA exchanged NCs. A) Signal observed at varying time delay. B) Integrated intensity decay with delay time. The data was fitted with equation 3 to yield a $T_{2}$ of 26.9 ms. Given the peak FWHM of $64.5 \mathrm{~Hz}$ we obtain a homogenous FWHM of $11.8 \mathrm{~Hz}$ and heterogeneous FWHM of $50.7 \mathrm{~Hz}$. C) Residual to the fit in B. 

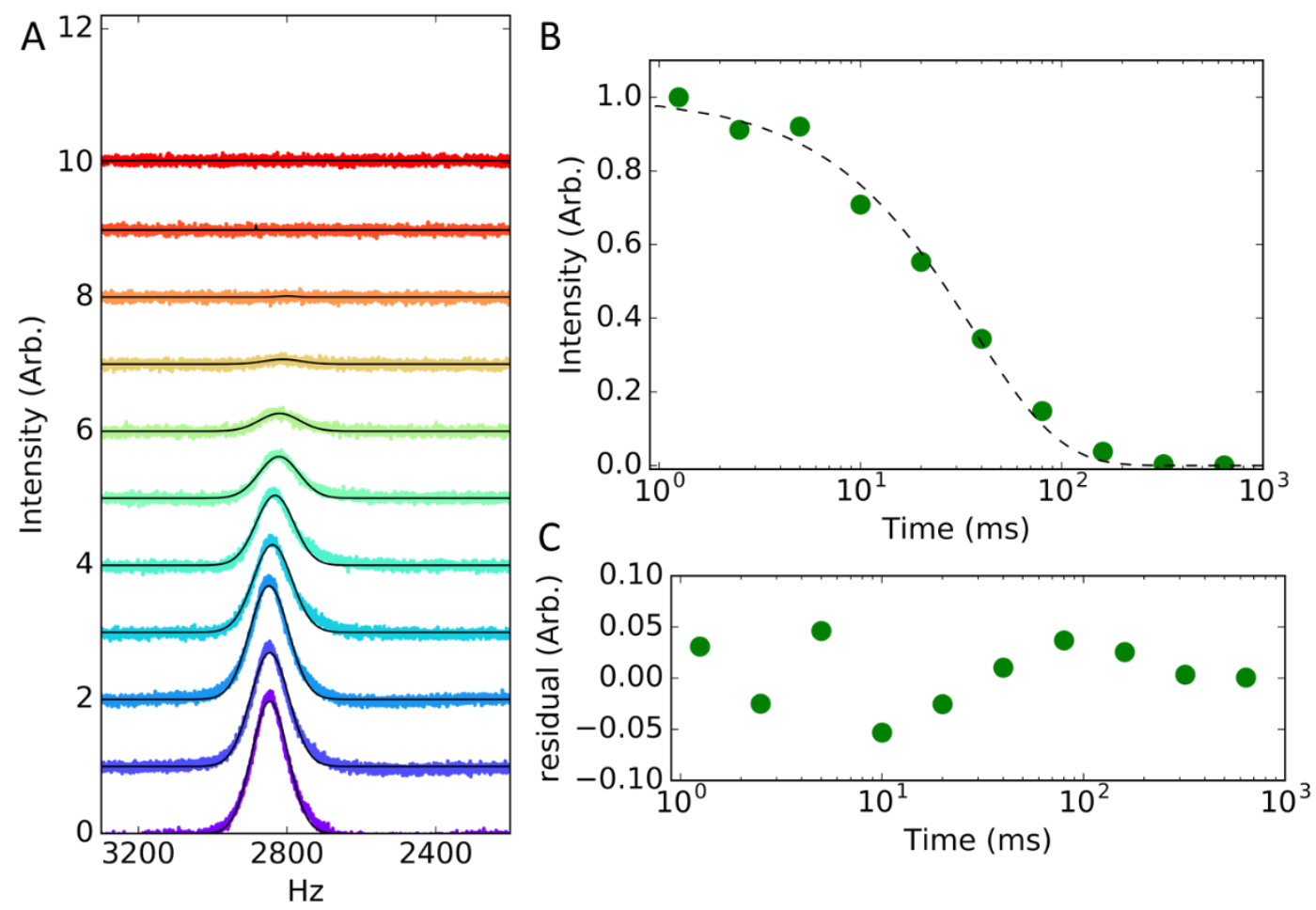

Figure S3: Linewidth analysis for 9-ACA exchanged NCs. A) Signal observed at varying time delay. B) Integrated intensity decay with delay time. The data was fitted with equation 3 to yield a $T_{2}$ of $36.35 \mathrm{~ms}$. Given the peak FWHM of $110.1 \mathrm{~Hz}$ we obtain a homogenous FWHM of $8.7 \mathrm{~Hz}$ and heterogeneous FWHM of $101.3 \mathrm{~Hz} . \mathrm{C}$ ) Residual to the fit in $\mathrm{B}$. 

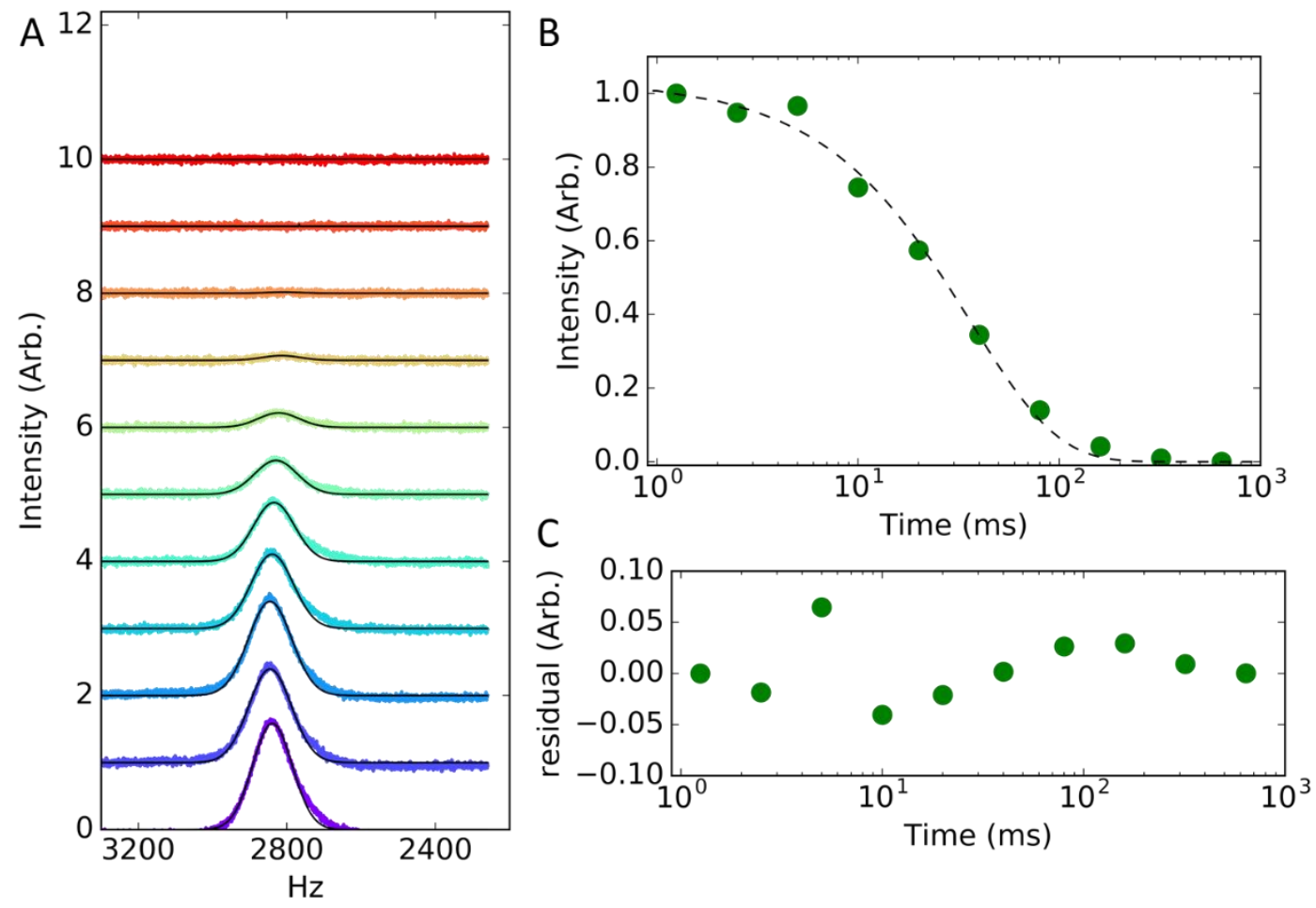

Figure S33: Linewidth analysis for 9-ACA exchanged NCs. A) Signal observed at varying time delay. B) Integrated intensity decay with delay time. The data was fitted with equation 3 to yield a $T_{2}$ of $36.17 \mathrm{~ms}$. Given the peak FWHM of $125.3 \mathrm{~Hz}$ we obtain a homogenous FWHM of $8.8 \mathrm{~Hz}$ and heterogeneous FWHM of $116.5 \mathrm{~Hz}$. C) Residual to the fit in B. 


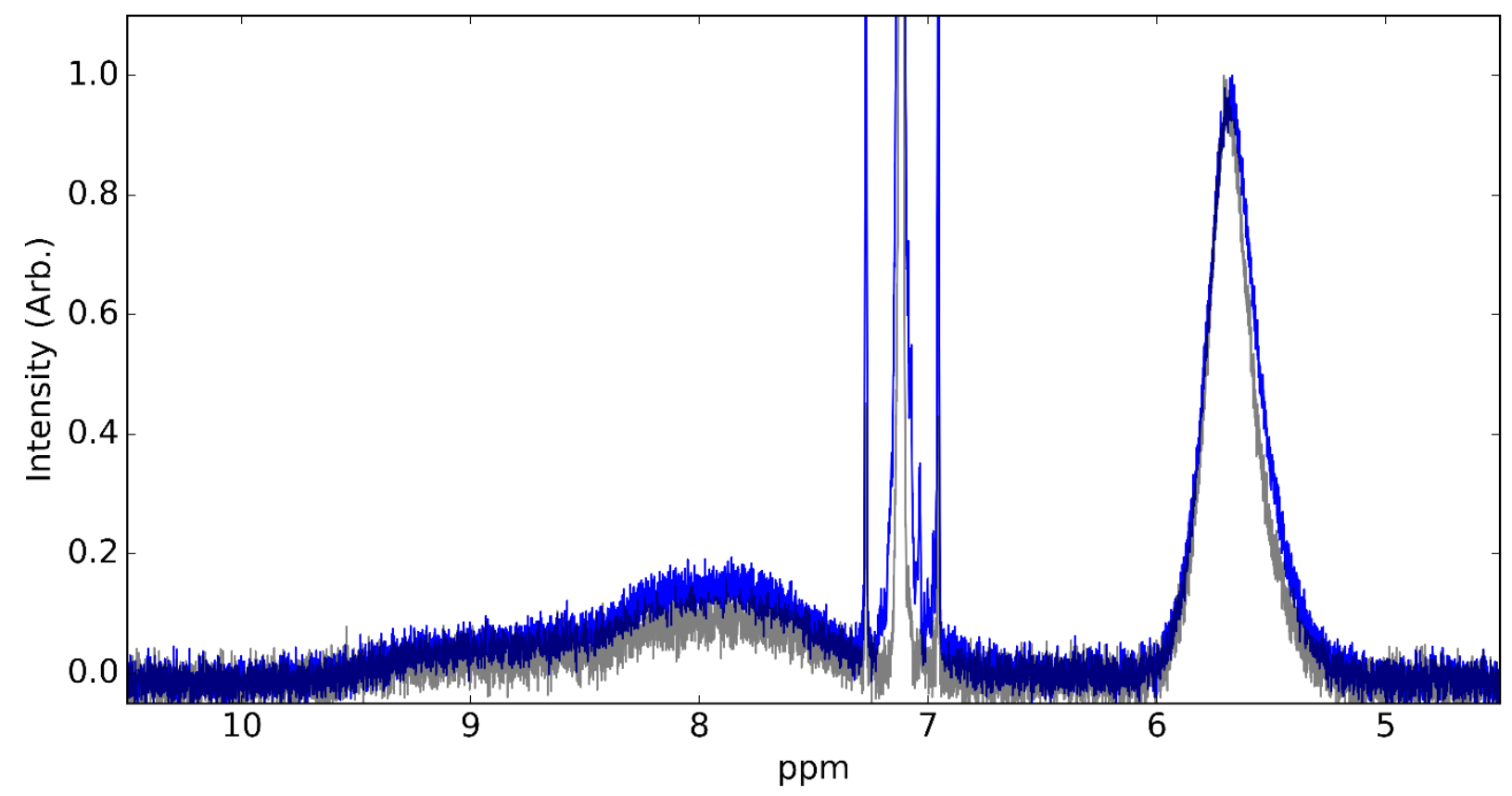

Figure S34: ${ }^{1} \mathrm{H}$ NMR spectra of the mixed 9-ACA/OA samples used to generate figures S29 (gray) and S30 (blue). We observe that a wider width of the vinylic protons of OA correlates with the amount of 9-ACA present on the NC. This was also observed in figures S8 to S12. 
Supplemental Section 8: Transmission Electron Microscopy characterization
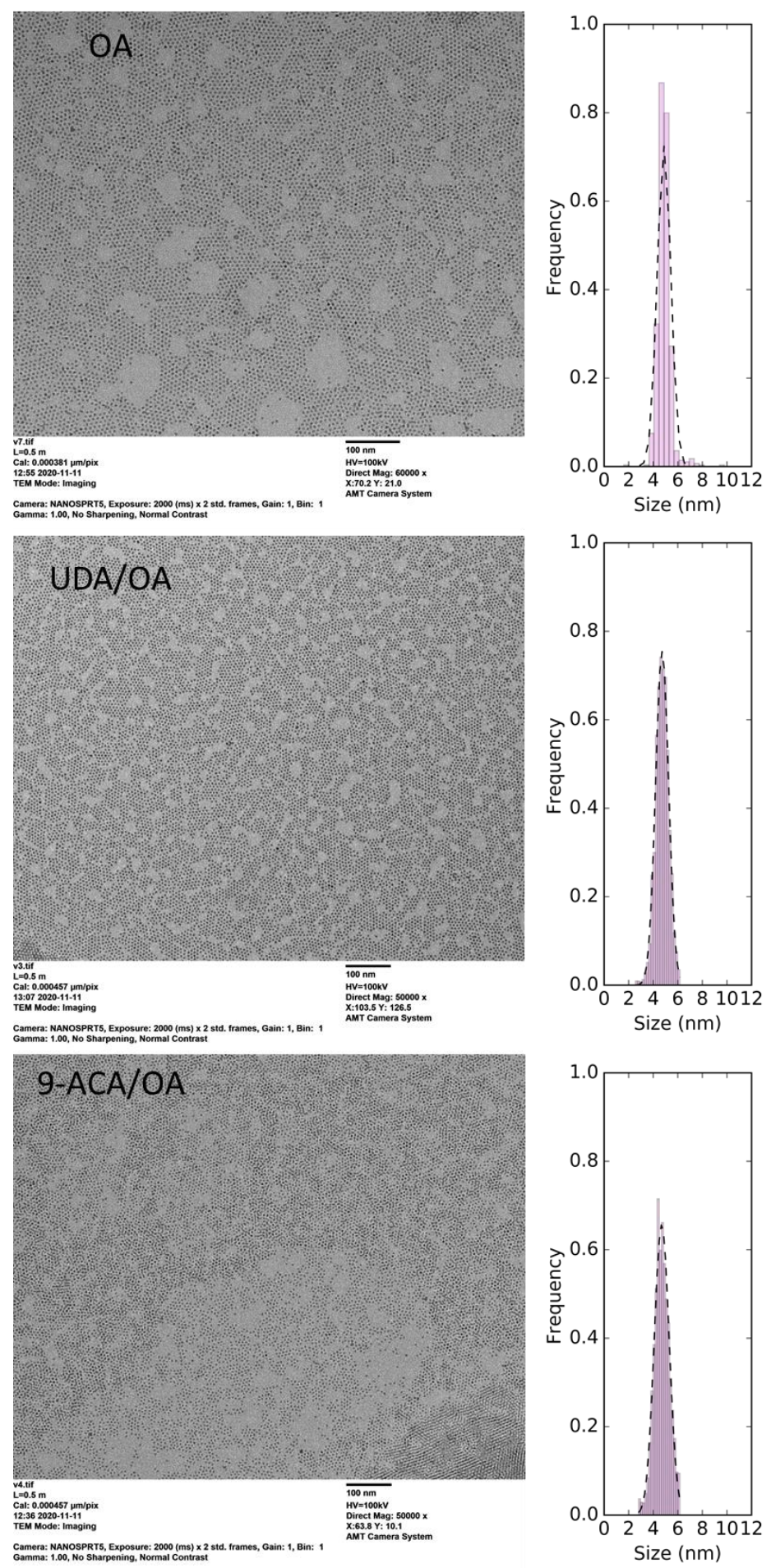

Figure S35: TEM micrograph and corresponding sizing histogram of native OA passivated NC (Top), UDA/OA NCs (middles) and 9-ACA/OA NCs (bottom). Information from sizing histograms are summarized in Table $\mathrm{S} 2$ below. 
Table S2: Calculated and estimated size and size dispersions of nanocrystals with different ligands

\begin{tabular}{lllll}
\hline Ligands & $\begin{array}{l}\text { Number of NCs } \\
\text { sized }\end{array}$ & Diameter $(\mathbf{n m})$ & $\begin{array}{l}\text { Standard deviation } \\
(\mathbf{n m})\end{array}$ & $\begin{array}{l}\text { TEM Size dispersion } \\
(\%)\end{array}$ \\
\hline OA & 680 & 4.88 & 0.55 & 11 \\
\hline 9-ACA/OA & 9943 & 4.73 & 0.65 & 14 \\
\hline UDA/OA & 14357 & 4.72 & 0.52 & 11 \\
\hline
\end{tabular}




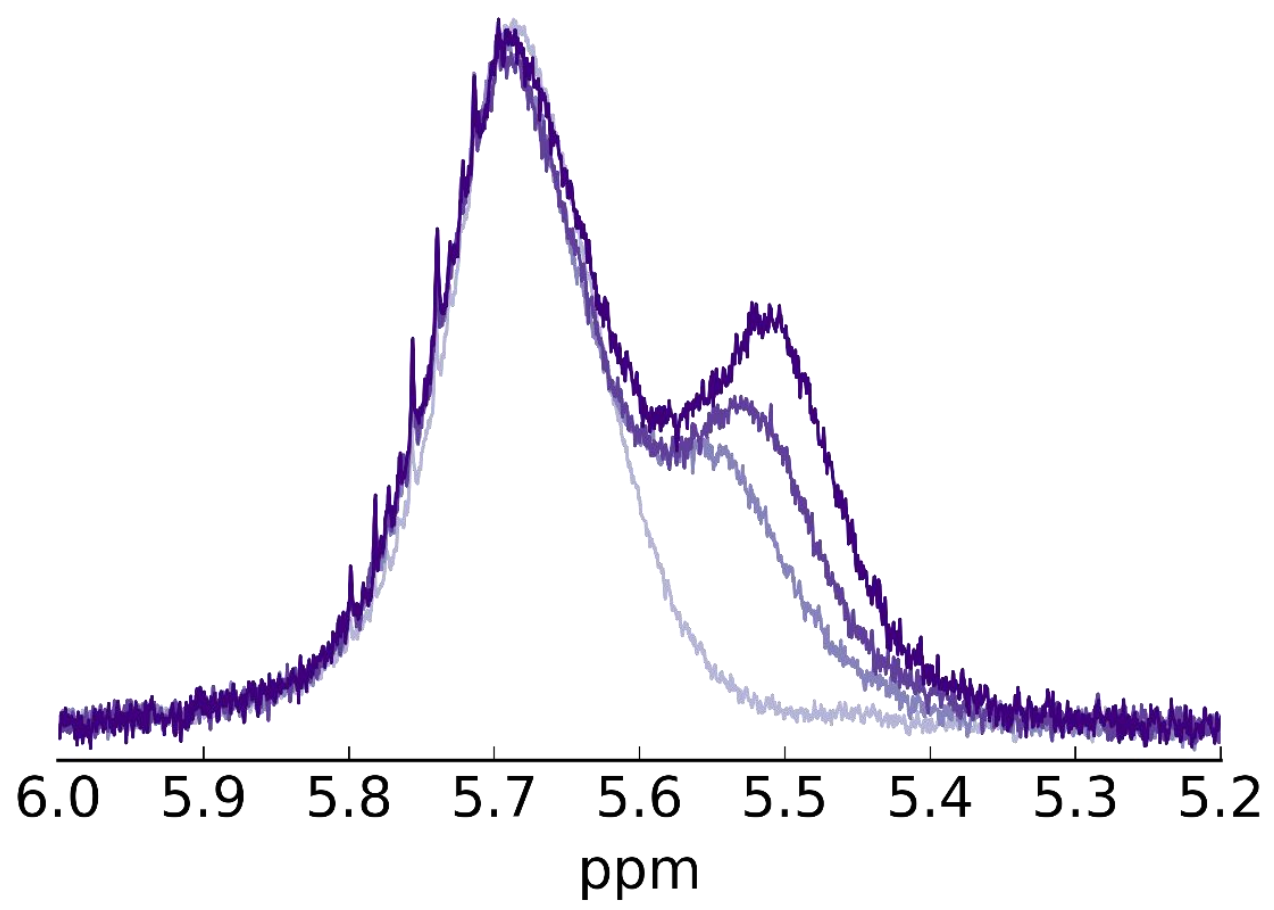

Figure S36: ${ }^{1} \mathrm{H}$ NMR of PbS NCs through X-for-X exchanges with 2-HDA. The initial peak (pale purple) near $5.7 \mathrm{ppm}$ is due to the bound vinylic protons of OA. As 2-HDA is titrated (darker purple) free OA is observed to appear around $5.5 \mathrm{ppm}$. This confirms that 2-HDA has been exchanged on the NC surface. All the peaks have been normalized to the bound OA signal to demonstrate that no peak broadening is observed throughout the titration. 


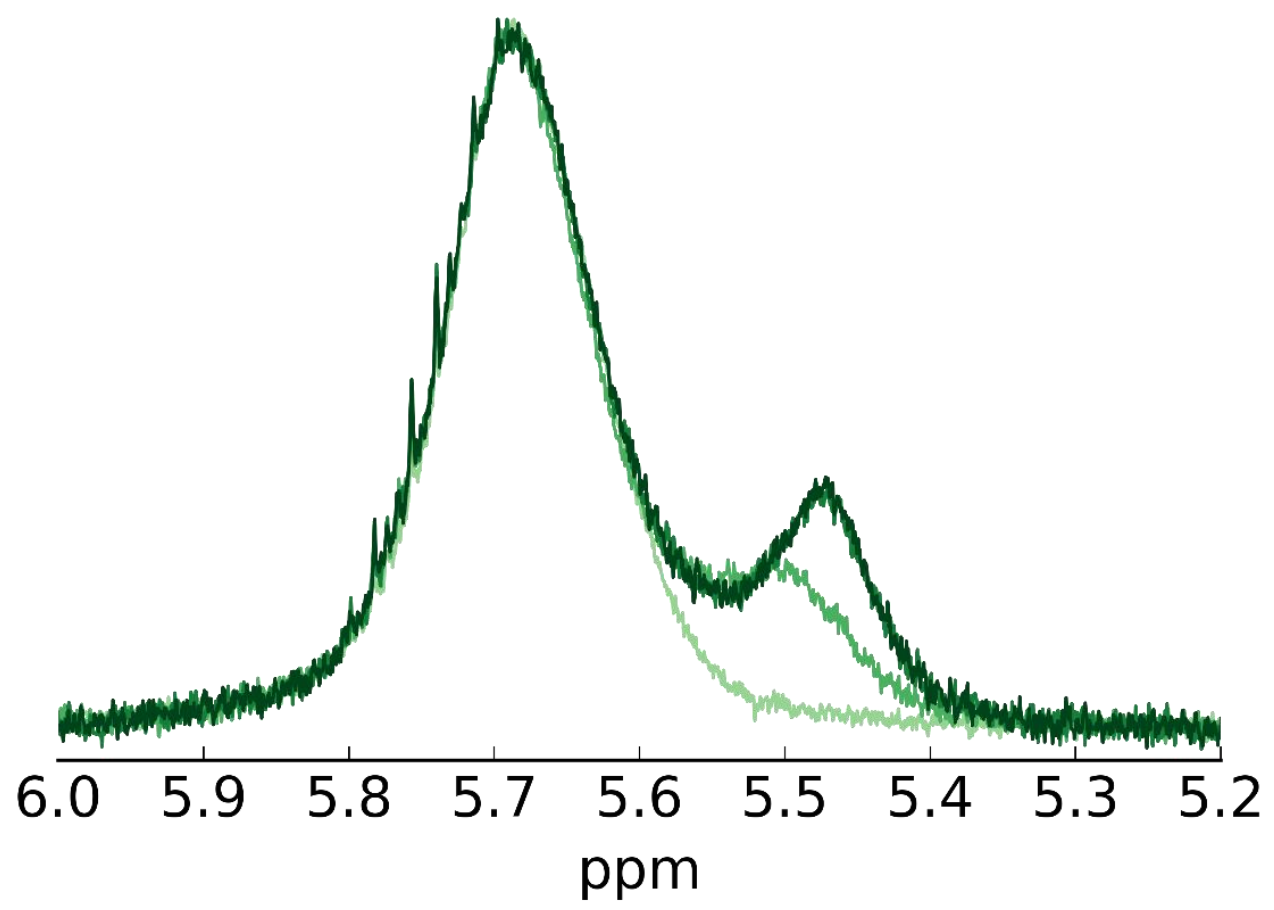

Figure S37: ${ }^{1} \mathrm{H}$ NMR of PbS NCs through X-for-X exchanges with DCHAA. The initial peak (pale green) near $5.7 \mathrm{ppm}$ is due to the bound vinylic protons of OA. As DCHAA is titrated (darker green) free OA is observed to appear around $5.5 \mathrm{ppm}$. This confirms that DCHAA has been exchanged on the NC surface. All the peaks have been normalized to the bound OA signal to demonstrate that no peak broadening is observed throughout the titration. 


\section{References:}

(1) Green, P. B.; Narayanan, P.; Li, Z.; Sohn, P.; Imperiale, C. J.; Wilson, M. W. B. Controlling Cluster Intermediates Enables the Synthesis of Small PbS Nanocrystals with Narrow Ensemble Line Widths. Chem. Mater. 2020, 32 (9), 4083-4094.

(2) Lee, J. W.; Kim, D. Y.; Baek, S.; Yu, H.; So, F. Photodetectors: Inorganic UV-Visible-SWIR Broadband Photodetector Based on Monodisperse PbS Nanocrystals (Small 10/2016). Small 2016, 12 (10), 1246-1246.

(3) Hines, M.; Scholes, G. D. Colloidal PbS Nanocrystals with Size-Tunable Near-Infrared Emission: Observation of Post-Synthesis Self-Narrowing of the Particle Size Distribution. Adv. Mater. 2003, 15 (21), 1844-1849.

(4) Moreels, I.; Lambert, K.; Smeets, D.; Muynck, D. De; Nollet, T.; Martins, C.; Vanhaecke, F.; Delerue, C.; Allan, G.; Hens, Z. Size-Dependent Optical Properties of Colloidal PbS Quantum Dots. ACS Nano 2009, 3 (10), 3023-3030.

(5) Kessler, M. L.; Starr, H. E.; Knauf, R. R.; Rountree, K. J.; Dempsey, J. L. Exchange Equilibria of Carboxylate-Terminated Ligands at PbS Nanocrystal Surfaces. Phys. Chem. Chem. Phys. 2018, 20 (36), 23649-23655.

(6) De Nolf, K.; Cosseddu, S. M.; Jasieniak, J. J.; Drijvers, E.; Martins, J. C.; Infante, I.; Hens, Z. Binding and Packing in Two-Component Colloidal Quantum Dot Ligand Shells: Linear versus Branched Carboxylates. J. Am. Chem. Soc. 2017, 139 (9), 3456-3464.

(7) Gomes, R.; Hassinen, A.; Szczygiel, A.; Zhao, Q.; Vantomme, A.; Martins, J. C.; Hens, Z. Binding of Phosphonic Acids to CdSe Quantum Dots: A Solution NMR Study. J. Phys. Chem. Lett. 2011, 2 (3), 145-152.

(8) Nemat, S. J.; Van den Eynden, D.; Deblock, L.; Heilmann, M.; Köster, J. M.; Parvizian, M.; Tiefenbacher, K.; De Roo, J. Resorcin[4]Arene-Based Multidentate Phosphate Ligands with Superior Binding Affinity for Nanocrystal Surfaces. Chem. Commun. 2021.

(9) Kroupa, D. M.; Anderson, N. C.; Castaneda, C. V.; Nozik, A. J.; Beard, M. C. In Situ Spectroscopic Characterization of a Solution-Phase X-Type Ligand Exchange at Colloidal Lead Sulphide Quantum Dot Surfaces. Chem. Commun. 2016, 52 (96), 13893-13896.

(10) Knauf, R. R.; Lennox, J. C.; Dempsey, J. L. Quantifying Ligand Exchange Reactions at CdSe Nanocrystal Surfaces. Chem. Mater. 2016, 28 (13), 4762-4770.

(11) Drijvers, E.; De Roo, J.; Martins, J. C.; Infante, I.; Hens, Z. Ligand Displacement Exposes Binding Site Heterogeneity on CdSe Nanocrystal Surfaces. Chem. Mater. 2018, 30 (3), 1178-1186.

(12) Saniepay, M.; Mi, C.; Liu, Z.; Abel, E. P.; Beaulac, R. Insights into the Structural Complexity of Colloidal CdSe Nanocrystal Surfaces: Correlating the Efficiency of Nonradiative Excited-State Processes to Specific Defects. J. Am. Chem. Soc. 2018, 140 (5), 1725-1736.

(13) Kessler, M. L.; Dempsey, J. L. Mapping the Topology of PbS Nanocrystals through Displacement Isotherms of Surface-Bound Metal Oleate Complexes. Chem. Mater. 2020, 32 (6), 2561-2571.

(14) Spano, F. C. The Spectral Signatures of Frenkel Polarons in H- And J-Aggregates. Acc. Chem. Res. 2010, 43 (3), 429-439. 
(15) Xia, P.; Raulerson, E. K.; Coleman, D.; Gerke, C. S.; Mangolini, L.; Tang, M. L.; Roberts, S. T. Achieving Spin-Triplet Exciton Transfer between Silicon and Molecular Acceptors for Photon Upconversion. Nat. Chem. 2020, 12 (2), 137-144.

(16) Wu, M.; Vartanian, A. M.; Chong, G.; Pandiakumar, A. K.; Hamers, R. J.; Hernandez, R.; Murphy, C. J. Solution NMR Analysis of Ligand Environment in Quaternary Ammonium-Terminated SelfAssembled Monolayers on Gold Nanoparticles: The Effect of Surface Curvature and Ligand Structure. J. Am. Chem. Soc. 2019, 141 (10), 4316-4327.

(17) De Roo, J.; Yazdani, N.; Drijvers, E.; Lauria, A.; Maes, J.; Owen, J. S.; Van Driessche, I.; Niederberger, M.; Wood, V.; Martins, J. C.; Infante, I.; Hens, Z. Probing Solvent-Ligand Interactions in Colloidal Nanocrystals by the NMR Line Broadening. Chem. Mater. 2018, 30 (15), 5485-5492. 